\title{
SPLITTING METHODS BASED ON ALGEBRAIC FACTORIZATION FOR FLUID-STRUCTURE INTERACTION*
}

\author{
SANTIAGO BADIA ${ }^{\dagger}$, ANNALISA QUAINI $^{\ddagger}$, AND ALFIO QUARTERONI ${ }^{\S}$
}

\begin{abstract}
We discuss in this paper the numerical approximation of fluid-structure interaction (FSI) problems dealing with strong added-mass effect. We propose new semi-implicit algorithms based on inexact block- $L U$ factorization of the linear system obtained after the space-time discretization and linearization of the FSI problem. As a result, the fluid velocity is computed separately from the coupled pressure-structure velocity system at each iteration, reducing the computational cost. We investigate explicit-implicit decomposition through algebraic splitting techniques originally designed for the FSI problem. This approach leads to two different families of methods which extend to FSI the algebraic pressure correction method and the Yosida method, two schemes that were previously adopted for pure fluid problems. Furthermore, we have considered the inexact factorization of the fluid-structure system as a preconditioner. The numerical properties of these methods have been tested on a model problem representing a blood-vessel system.
\end{abstract}

Key words. fluid-structure interaction, semi-implicit coupling, inexact factorization, algebraic splitting methods, added-mass effect, blood flow

AMS subject classifications. 74F10, 65N30, 76D05

DOI. $10.1137 / 070680497$

1. Introduction. We are interested in the numerical approximation of the heterogeneous mechanical system which couples the equations governing a fluid flow and the deformation of a structure; this situation arises in many engineering problems.

A great variety of strategies have been proposed to solve fluid-structure interaction (FSI) problems. A first issue is how to deal with the nonlinearity of the problem. In fact, not only are the fluid (and in some cases the structure) equations nonlinear, but the structure displacement also modifies the fluid domain generating geometrical nonlinearities. The fixed point technique (e.g., [3, 20, 21, 10]) is the simplest to linearize the FSI problem; however, Newton (e.g., [13]) and quasi-Newton (e.g., [30, $15,19,17])$ methods have also been considered.

A classical restriction for fluid-structure algorithms is modularity. Most of the time the codes for the pure fluid problem and for the pure structure problem already exist, and they are optimized for the specific mathematical features of the two different problems. Then the best way to solve the FSI problem would be to design algorithms

${ }^{*}$ Received by the editors January 19, 2007; accepted for publication (in revised form) December 17, 2007; published electronically April 23, 2008.

http://www.siam.org/journals/sisc/30-4/68049.html

†Universitat Politècnica de Catalunya, International Center for Numerical Methods in Engineering (CIMNE), Jordi Girona 1-3, Edifici C1, 08034 Barcelona, Spain (sbadia@cimne.upc.edu, sibadia@sandia.gov). Most of this work has been carried out during a postdoctoral stay at MOX supported by the European Community through the Research Training Network "Mathematical Modelling of the Cardiovascular System (HaeMOdel)," contract HPRN-CT-2002-00270. This author's work was also supported by the European Commission through FP6 project DISHEART (COOPCT-2004-513226) and by the Ministerio de Educación y Ciencia of Spain through Plan Nacional $\mathrm{I}+\mathrm{D}+\mathrm{i}$, project SIMCV DPI2004-07410-C03-02.

${ }^{\ddagger}$ IACS-Chair of Modeling and Scientific Computing, EPFL, CH-1015 Lausanne, Switzerland (annalisa.quaini@epfl.ch). The work of this author was supported by the Swiss National Science Foundation under grant 200021 - 109378/1.

$\S$ MOX-Dipartimento di Matematica "F. Brioschi," Politecnico di Milano, I-20133 Milano, Italy (alfio.quarteroni@epfl.ch). 
involving only communication between the codes. Substructuring techniques stemming from a domain decomposition viewpoint $[9,8]$ well serve this purpose. Among these procedures, the classical Dirichlet-Neumann technique is one of the most widely used. Usually, iterations are carried out by using Richardson or conjugate gradient methods for the interface equation.

The load exerted by the fluid on the structure can be interpreted as an added-mass [2]. When the structure density is much bigger than the fluid density, as it happens in aeroelasticity, the added-mass effect is negligible, and the numerical approximation of the FSI problem through iterative procedures is less challenging. However, when the two densities are of the same order of magnitude, as in hemodynamics, the added-mass effect becomes important, and iterative procedures fail or are too slow. Richardson coupling iterations become ineffective, and more elaborate techniques, such as GMRES or BiCG, are required. These iterative procedures are minimization techniques that always converge, but their convergence rate will depend on the relevance of the added-mass effect.

Another option is to solve the monolithic fluid-structure system (after linearization and discretization). Lack of modularity is the main drawback of this approach. In fact, it does not allow one to reuse existing codes for the two subproblems and requires the development of a global FSI solver. We refer to [17] for the solution of this monolithic system using multigrid techniques.

An alternative to substructuring techniques and monolithic methods is given by semi-implicit schemes, introduced in [12]. The idea is to decouple the fluid velocty
computation from the strongly coup ed fluil-structure system, which involves orly
pressure and structure unknowns, with the double advantage of reducing computa-
tional costs and ensuring stability. In fact, since the pressure is still coupled to the
structure, the stability of the schmes is independent of the added-mas effect. In
[12], the FSI system is sdlved through the Chorin-Temam proiection scheme [4 29].

In this paper, we apply the same kind of explicit-implicit splitting, but we derive it

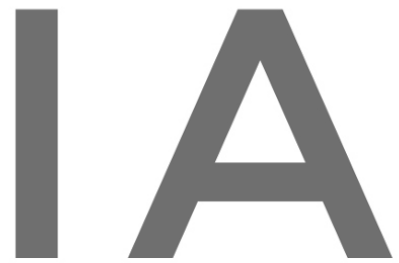

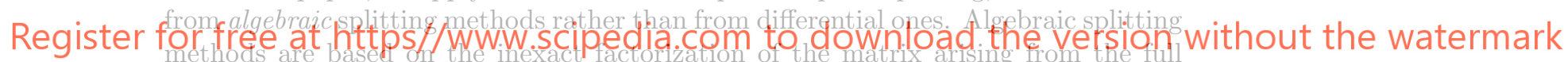

discretization (in both time and space) of the given initial-boundary value problem. In this way, the boundary conditions are already incorporated in the discretized operator, and no further boundary conditions have to be selected. Our aim is to take advantage of the good accuracy properties shown by many of these techniques (which do not have a differential counterpart $[25,26,16]$ ) when solving the incompressible Navier-Stokes equations. In this respect, this work develops and generalizes the approach proposed in [23]. In particular, we present the incremental version of the Yosida method for FSI problems, whose nonincremental version was introduced in [23], and we adapt the algebraic pressure-correction methods to the coupled fluid-structure problem.

Finally, we propose to use the inexact factorization of the FSI system matrix as a preconditioner, leading to predictor-corrector methods that converge to the solution of the monolithic FSI system.

The outline of the paper is the following. In section 2, we state the FSI problem in its strong and weak form and tackle its space-time discretization and linearization. Section 3 (the core of the article) is devoted to inexact factorization techniques for the FSI system, which lead to two families of semi-implicit methods. Then we consider the inexact factorization of the fluid-structure system as a preconditioner. In section 4 , we compare the new methods with the semi-implicit coupling scheme proposed in [12]. Section 5 contains the analysis of the perturbation error due to the inexact factorization. In section 6 , we suggest some methodologies for the solution of the 
pressure-structure problem. We analyze the numerical properties of the new algorithms for a test case that consists of a bidimensional blood-vessel system in section 7. Finally, some conclusions are drawn in section 8.

2. Problem setting. Consider an heterogeneous mechanical system which covers a bounded, polyhedral, and moving domain $\Omega_{t} \subset \mathbb{R}^{d}(d=2,3$ being the space dimension), where time $t$ belongs to the interval of analysis $[0, T]$. This domain is divided into a domain $\Omega_{t}^{s}$ occupied by a solid structure and its complement $\Omega_{t}^{f}$ occupied by the fluid. The fluid-structure interface $\Sigma_{t}$ is the common boundary between $\Omega_{t}^{f}$ and $\Omega_{t}^{s}$, i.e., $\Sigma_{t}=\partial \Omega_{t}^{f} \cap \partial \Omega_{t}^{s}$. Furthermore, $\boldsymbol{n}_{f}$ is the outward normal of $\Omega_{t}^{f}$ on $\Sigma_{t}$, and $\boldsymbol{n}_{s}$ is its counterpart for the structure domain. The initial configuration $\Omega_{0}$ at $t=0$ is considered as the reference one.

In order to describe the evolution of the whole domain $\Omega_{t}$, we define two families of mappings:

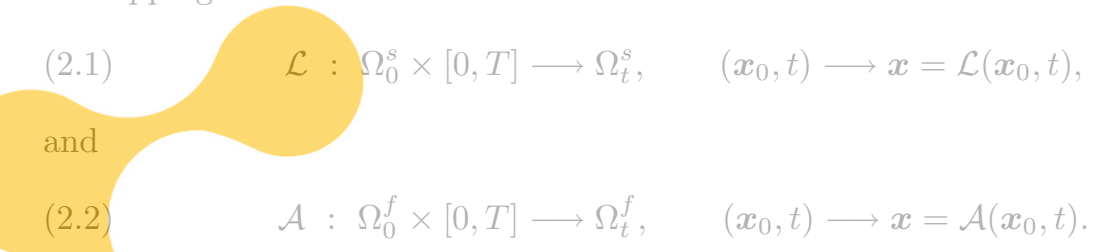

The map $\mathcal{L}_{t}=\mathcal{L}(\cdot, t)$ tracks the solid domain in time $\mathcal{A}_{t}=\mathcal{A}(\cdot, t)$ the fluid domain, and they must agree on

$(2.3) \quad(2)$

$(2.3)$

in order to define an hom

We adopt a purely Lagrangian
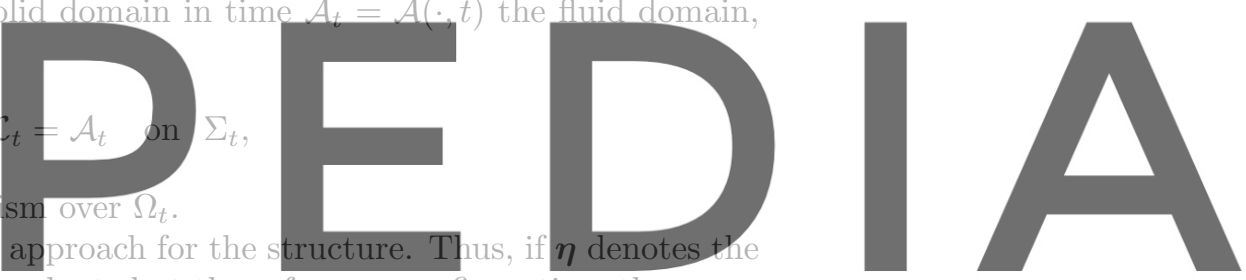

displacement of the solid medium evaluated at the reference configuration, then

\section{Register for free at https//www.scipedia,camatø).download the version without the watermark}

Apart from (2.3), the fluid domain mapping $\mathcal{A}_{t}$ is arbitrary. This mapping can be defined as an appropriate extension operator of its value on the interface:

$$
\mathcal{A}_{t}\left(\boldsymbol{x}_{0}\right)=\boldsymbol{x}_{0}+\operatorname{Ext}\left(\left.\boldsymbol{\eta}\left(\boldsymbol{x}_{0}, t\right)\right|_{\Sigma_{0}}\right) .
$$

A classical choice is to consider a harmonic extension in the reference domain. $\mathcal{A}_{t}$ is called the arbitrary Lagrangian-Eulerian (ALE) mapping, since in general it does not track the fluid particles (in that case the formulation would be purely Lagrangian).

For any function $f: \Omega_{t} \times[0, T] \longrightarrow \mathbb{R}$, we indicate with $\hat{f}=f \circ \mathcal{A}_{t}$ the corresponding function in the ALE frame:

$$
\hat{f}: \Omega_{0} \times[0, T] \longrightarrow \mathbb{R}, \quad \hat{f}\left(\boldsymbol{x}_{0}, t\right)=f\left(\mathcal{A}_{t}\left(\boldsymbol{x}_{0}\right), t\right) .
$$

The time derivatives in the ALE frame are defined as follows:

$$
\left.\partial_{t} f\right|_{\boldsymbol{x}_{0}}: \Omega_{t} \times[0, T] \longrightarrow \mathbb{R},\left.\quad \partial_{t} f\right|_{\boldsymbol{x}_{0}}(\boldsymbol{x}, t)=\partial_{t} \hat{f} \circ \mathcal{A}_{t}^{-1}(\boldsymbol{x}) .
$$

The domain velocity $\boldsymbol{w}$ is calculated by using the following expression:

$$
\boldsymbol{w}(\boldsymbol{x}, t)=\left.\partial_{t} \boldsymbol{x}\right|_{\boldsymbol{x}_{0}}=\partial_{t} \mathcal{A}_{t} \circ \mathcal{A}_{t}^{-1}(\boldsymbol{x}) .
$$


Then, owing to (2.4):

$$
\hat{\boldsymbol{w}}\left(\boldsymbol{x}_{0}, t\right)=\operatorname{Ext}\left(\left.\partial_{t} \boldsymbol{\eta}\left(\boldsymbol{x}_{0}, t\right)\right|_{\Sigma_{0}}\right) .
$$

The solid is assumed to be a hyperelastic material, characterized by a constitutive law relating the stress tensor $\boldsymbol{\sigma}^{s}$ to $\boldsymbol{\eta}$. We assume the fluid to be homogeneous, Newtonian, and incompressible. We indicate with $\boldsymbol{\sigma}^{f}$ its Cauchy stress tensor:

$$
\boldsymbol{\sigma}^{f}(\boldsymbol{u}, p)=-p \mathbf{I}+2 \mu \boldsymbol{\epsilon}(\boldsymbol{u}),
$$

where $p$ is the pressure, $\mu$ is the fluid viscosity, and

$$
\boldsymbol{\epsilon}(\boldsymbol{u})=\frac{1}{2}\left(\nabla \boldsymbol{u}+(\nabla \boldsymbol{u})^{T}\right)
$$

is the strain rate tensor, with $\nabla$ denoting the spatial gradient operator.

In order to write the fluid problem in its ALE form, let us apply the chain rule to the velocity time derivative:

$(2.6)$

$$
\left.\partial_{t} \boldsymbol{u}\right|_{x_{0}}=\partial_{t} \boldsymbol{u}+\boldsymbol{w} \cdot \nabla \boldsymbol{u},
$$

where $\partial_{t} u$ is the partial time derivative in the spatial frame (Eulerian derivative).

The fluid-structure problem we will consider couples the incompressible Navier Stokes equations in the $A$ solid. We state this coup (those on the physical bou script $\hat{?}$ when by the co configuration. Thus, the

1. Geometry problem: Find
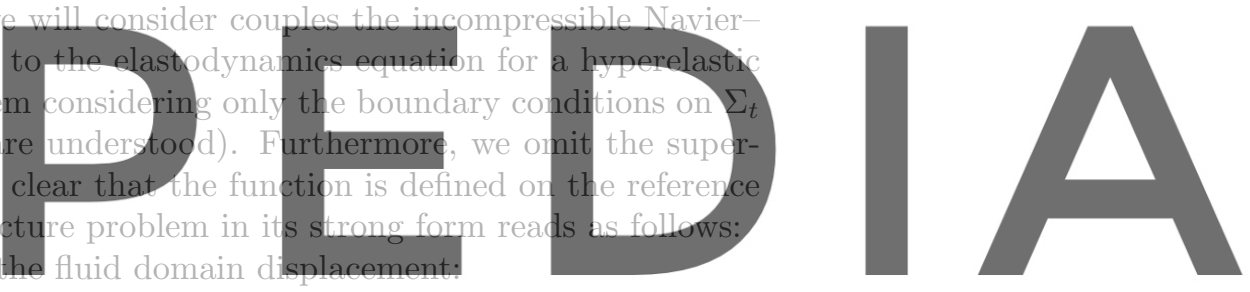

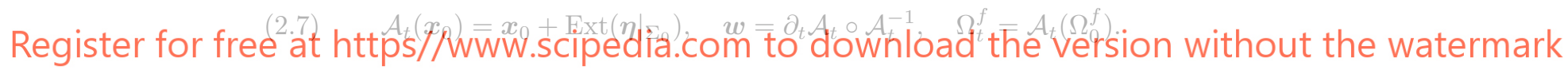

2. Fluid-structure problem: Find velocity $u$, pressure $p$, and displacement $\eta$ such that

$$
\begin{array}{lc}
\left.\partial_{t} \boldsymbol{u}\right|_{\boldsymbol{x}_{0}}+(\boldsymbol{u}-\boldsymbol{w}) \cdot \nabla \boldsymbol{u}-\frac{1}{\rho_{f}} \nabla \cdot \boldsymbol{\sigma}^{f}=\boldsymbol{f}_{f} & \text { in } \Omega_{t}^{f} \times(0, T), \\
\nabla \cdot \boldsymbol{u}=0 & \text { in } \Omega_{t}^{f} \times(0, T), \\
\partial_{t}^{2} \boldsymbol{\eta}-\frac{1}{\rho_{s}} \nabla \cdot \boldsymbol{\sigma}^{s}=\boldsymbol{f}_{s} & \text { in } \Omega_{t}^{s} \times(0, T), \\
\boldsymbol{u}=\partial_{t} \boldsymbol{\eta} & \text { on } \Sigma_{t} \times(0, T), \\
\boldsymbol{\sigma}^{s} \cdot \boldsymbol{n}_{s}+\boldsymbol{\sigma}^{f} \cdot \boldsymbol{n}_{f}=0 & \text { on } \Sigma_{t} \times(0, T) .
\end{array}
$$

Two transmission conditions are enforced at the interface: the continuity of fluid and structure velocities (2.8d), due to the adherence condition, and the continuity of stresses (2.8e), expressing the action-reaction principle. These two problems are also coupled by the geometrical condition (2.3).

2.1. Weak formulation. For the variational formulation of the fluid-structure problem (2.7)-(2.8), we indicate with $L^{2}(\Omega)$ the space of square integrable functions in a spatial domain $\Omega$, with $H^{1}(\Omega)$ the space of functions in $L^{2}(\Omega)$ with first derivatives in $L^{2}(\Omega)$ and with $H^{1}(\operatorname{div} ; \Omega)$ the space of functions whose divergence belongs to 
$L^{2}(\Omega)$. We use $(\cdot, \cdot)_{\Omega}$ and $\langle\cdot, \cdot\rangle_{\Omega}$ to denote the $L^{2}$ product and a duality pair in $\Omega$, respectively.

From now on we will consider a $(d-1)$-dimensional structure, and thus $\overline{\Omega_{t}^{s}}=\Sigma_{t}$. All of the results we will find in the following can, however, be generalized, with minor adaptations, to the case of $d$-dimensional structures (see Remarks 2 and 6 ). Let us define the following spaces, for any given $t \in[0, T)$ :

$$
\begin{aligned}
V^{f}(t) & :=\left\{\boldsymbol{v}: \Omega_{t}^{f} \rightarrow \mathbb{R}^{d}, \boldsymbol{v}=\hat{\boldsymbol{v}} \circ\left(\mathcal{A}_{t}\right)^{-1}, \hat{\boldsymbol{v}} \in\left(H^{1}\left(\Omega_{0}^{f}\right)\right)^{d}\right\}, \\
V_{0}^{f}(t) & :=\left\{\boldsymbol{v} \in V^{f}(t),\left.\boldsymbol{v}\right|_{\Sigma_{t}}=\mathbf{0}\right\}, \\
Q(t) & :=\left\{q: \Omega_{t}^{f} \rightarrow \mathbb{R}, q=\hat{q} \circ\left(\mathcal{A}_{t}\right)^{-1}, \hat{q} \in L^{2}\left(\Omega_{0}^{f}\right)\right\}, \\
\hat{V^{s}} & :=\left\{\hat{v}: \Omega_{0}^{s} \rightarrow \mathbb{R}^{d-1}, \hat{v} \in\left(H^{1}\left(\Omega_{0}^{s}\right)\right)^{d-1}\right\} .
\end{aligned}
$$

$(2.9)$

We write the convective term by using the following notation:

$(2.10)$

$$
c(\boldsymbol{u}, \boldsymbol{v}, \boldsymbol{w})_{\Omega_{t}^{f}}:=\rho_{f} \int_{\Omega_{t}^{f}}(\boldsymbol{u} \cdot \nabla \boldsymbol{v}) \cdot \boldsymbol{w} \mathrm{d} \Omega .
$$
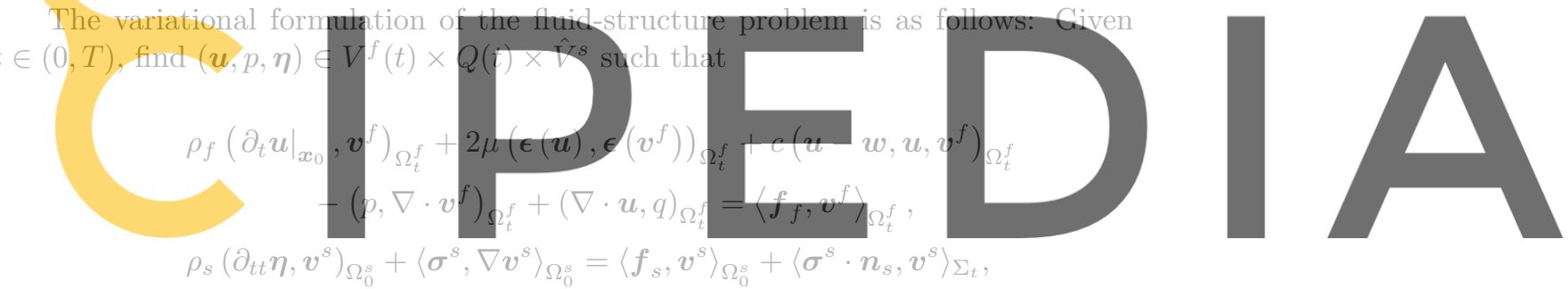

Register for free at lottos

for all $\left(v^{f}, q, v^{s}\right) \in V_{0}^{f}(t) \times Q(t) \times \hat{V}^{s}$. The continuity of velocities has been enforced in a strong way by (2.11). On the contrary, the continuity of stresses on the interface is satisfied in a weak way by choosing test functions $\boldsymbol{v}^{f} \in V^{f}(t)$ for the momentum conservation equation of the fluid problem. In fact, the fluid interface load can be seen as the variational residual of the weak form of the momentum conservation equation for test functions that do not vanish on $\Sigma_{t}$ :

$$
\begin{aligned}
\left\langle\boldsymbol{\sigma}^{f} \cdot \boldsymbol{n}_{f}, \boldsymbol{v}^{f}\right\rangle_{\Sigma_{t}}= & \rho_{f}\left(\left.\partial_{t} \boldsymbol{u}\right|_{\boldsymbol{x}_{0}}, \boldsymbol{v}^{f}\right)_{\Omega_{t}^{f}}+2 \mu\left(\boldsymbol{\epsilon}(\boldsymbol{u}), \boldsymbol{\epsilon}\left(\boldsymbol{v}^{f}\right)\right)_{\Omega_{t}^{f}}+c\left(\boldsymbol{u}-\boldsymbol{w}, \boldsymbol{u}, \boldsymbol{v}^{f}\right)_{\Omega_{t}^{f}} \\
& -\left(p, \nabla \cdot \boldsymbol{v}^{f}\right)_{\Omega_{t}^{f}}-\left\langle\boldsymbol{f}_{f}, \boldsymbol{v}^{f}\right\rangle_{\Omega_{t}^{f}} \\
= & :-\left\langle\mathcal{R}(\boldsymbol{u}, p), \boldsymbol{v}^{f}\right\rangle_{\Omega_{t}^{f}}
\end{aligned}
$$

Therefore, (2.8e) and (2.12) lead to the following equality:

$$
\left\langle\boldsymbol{\sigma}^{f} \cdot \boldsymbol{n}_{f}, \boldsymbol{v}^{s}\right\rangle_{\Sigma_{t}}=-\left\langle\mathcal{R}(\boldsymbol{u}, p), \mathcal{E}_{t}\left(\boldsymbol{v}^{s}\right)\right\rangle_{\Omega_{t}^{f}}
$$

for all $\boldsymbol{v}^{s} \in V^{s}, \mathcal{E}_{t}$ being an arbitrary extension operator $\mathcal{E}_{t}: \widehat{V}^{s} \longrightarrow V^{f}(t)$. 
The weak transmission of the fluid loads at the interface is crucial when carrying out stability and convergence analysis.

2.2. The fully discrete problem: Space and time discretization. Let us consider a family of quasi-uniform finite element partitions $\mathcal{T}_{h}(t)$ (see, e.g., [27]) defined, for every $t>0$, by the partition at the reference fluid configuration $\hat{\mathcal{T}}_{h}$ and the discrete ALE mapping $\mathcal{A}_{t}$, i.e., by abusing notation, $\mathcal{T}_{h}(t)=\mathcal{A}_{t}\left(\hat{\mathcal{T}}_{h}\right)$. As usual, $h$ represents the maximum size of the elements of $\mathcal{T}_{h}$. $\mathcal{T}_{h}$ induces a partition $\mathcal{I}_{h}$ of $\Sigma_{t}$. Let $\mathcal{I}_{H}$ be an independent partition of $\Sigma_{t}$ for the structure problem whose diameter is $H$. Thus, $h$ and $H$ refer to the level of refinement of the two partitions. For the sake of clarity, from now on we consider the case of matching grids on $\Sigma_{t}$, that is, $\mathcal{I}_{H} \equiv \mathcal{I}_{h}$.

Let $\hat{V}_{h}^{f} \subset\left[H^{1}\left(\Omega_{0}^{f}\right)\right]^{d}, \hat{V}_{0, h}^{f} \subset\left[H_{0}^{1}\left(\Omega_{0}^{f}\right)\right]^{d}, \hat{Q}_{h}^{f} \subset L^{2}\left(\Omega_{0}^{f}\right)$, and $\hat{V}_{h}^{s} \subset\left[H^{1}\left(\Omega_{0}^{s}\right)\right]^{d-1}$ be the finite element spaces approximating $V^{f}, V_{0}^{f}, Q$, and $\hat{V}^{s}$ at the reference configuration, respectively. Again with an abuse of notation, we can define the finite element spaces for a given time step $t^{n}$ by using the domain maps (2.1)-(2.2), e.g., $V_{h}^{f}\left(t^{n}\right)=\mathcal{A}_{t^{n}}\left(\hat{V}_{h}^{f}\right)$. From now on we omit the time label $t^{n}$ from the finite element spaces names. We introduce the Lagrange basis $\left\{\phi_{i}\right\}_{\mathcal{N}_{f}} \oplus\left\{\phi_{j}^{\sigma}\right\}_{\mathcal{N}_{\sigma}},\left\{\pi_{i}\right\}_{\mathcal{N}_{p}}$, and $\left\{\boldsymbol{\psi}_{i}\right\}_{\mathcal{N}_{s}}$ associated to $V_{h}^{f}, Q_{h}^{f}$, and $\hat{V}_{h}^{s}$, respectively. $\mathcal{N}_{\sigma}$ denotes the set of velocity nodes on $\Sigma$, and $\mathcal{N}_{f}$ denotes the rest of the velocity nodes. The sets of pressure and
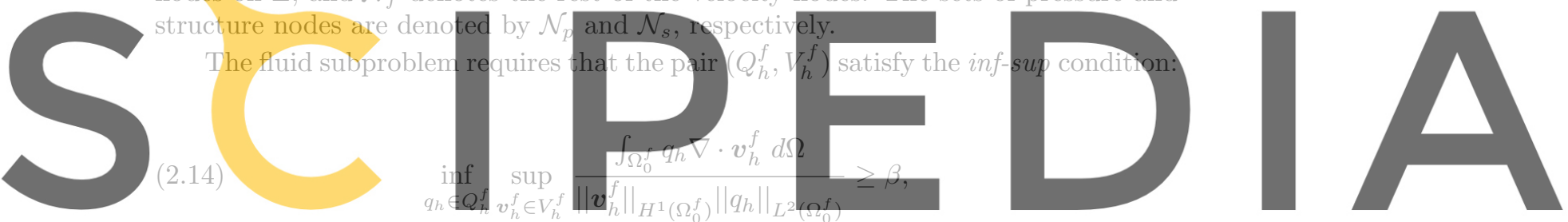

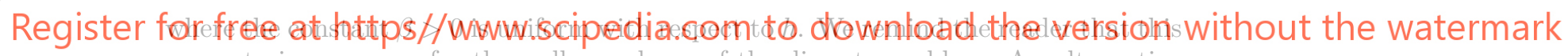

property is necessary for the well posedness of the discrete problem. An alternative to using inf-sup stable pairs is to resort to stabilization techniques: They modify the discrete problem so that it is stable for equal order velocity-pressure interpolations. In this work, we consider inf-sup stable elements even though all of the schemes suggested can be easily extended to the case of pressure-stabilized methods. For the stabilization of the convective term for high Reynolds numbers, we consider the orthogonal subgrid scales technique (see [5]). We denote by $c_{h}$ the trilinear form that stands for the convective term and stabilization terms.

With regard to time discretization, we have considered the backward Euler scheme for the fluid equations and the midpoint rule for the structure [28] for simplicity. In any case, the splitting methods suggested below can be easily extended to other time integration schemes (see Remark 1). By defining the backward Euler operator $\delta_{t}$ as $\delta_{t} f^{n+1}=\left(f^{n+1}-f^{n}\right) / \delta t$ and denoting by $\operatorname{Ext}_{h}(\cdot)$ a discretized version of the extension operator $\operatorname{Ext}(\cdot)$, at each time level $t^{n+1}$, the fully discretized fluid-structure problem reads:

1. Geometry problem: Find the fluid domain displacement

$$
\begin{aligned}
\mathcal{A}_{t^{n+1}}\left(\boldsymbol{x}_{0}\right) & =\boldsymbol{x}_{0}+\operatorname{Ext}_{h}\left(\left.\boldsymbol{\eta}_{h}^{n+1}\right|_{\Sigma_{0}}\right), \\
\boldsymbol{w}_{h}^{n+1} & =\delta_{t} \mathcal{A}_{t^{n+1}} \circ \mathcal{A}_{t^{n+1}}^{-1}, \quad \Omega_{t^{n+1}}^{f}=\mathcal{A}_{t^{n+1}}\left(\Omega_{0}^{f}\right) .
\end{aligned}
$$


2. Fluid-structure problem: Find $\left(\boldsymbol{u}_{h}^{n+1}, p_{h}^{n+1}, \boldsymbol{\eta}_{h}^{n+1}\right) \in V_{h}^{f} \times Q_{h} \times \hat{V}_{h}^{s}$ such that

$$
\begin{aligned}
\rho_{f}\left(\left.\delta_{t} \boldsymbol{u}_{h}^{n+1}\right|_{\boldsymbol{x}_{0}}, \boldsymbol{v}_{h}^{f}\right)_{\Omega_{t^{n+1}}^{f}}+2 \mu\left(\boldsymbol{\epsilon}\left(\boldsymbol{u}_{h}^{n+1}\right), \boldsymbol{\epsilon}\left(\boldsymbol{v}_{h}^{f}\right)\right)_{\Omega_{t^{n+1}}^{f}} \\
+c_{h}\left(\boldsymbol{u}_{h}^{n+1}-\boldsymbol{w}_{h}^{n+1}, \boldsymbol{u}_{h}^{n+1}, \boldsymbol{v}_{h}^{f}\right)_{\Omega_{t^{n+1}}^{f}}-\left(p_{h}^{n+1}, \nabla \cdot \boldsymbol{v}_{h}^{f}\right)_{\Omega_{t^{n+1}}^{f}} \\
\quad+\left(\nabla \cdot \boldsymbol{u}_{h}^{n+1}, q_{h}\right)_{\Omega_{t^{n+1}}^{f}}=\left\langle\boldsymbol{f}_{f}^{n+1}, \boldsymbol{v}_{h}^{f}\right\rangle_{\Omega_{t^{n+1}}^{f}},
\end{aligned}
$$

$$
\rho_{s}\left(\frac{\dot{\boldsymbol{\eta}}_{h}^{n+1}-\dot{\boldsymbol{\eta}}_{h}^{n}}{\delta t}, \boldsymbol{v}_{h}^{s}\right)_{\Omega_{0}^{s}}+\left\langle\boldsymbol{\sigma}^{s}\left(\frac{\boldsymbol{\eta}_{h}^{n+1}+\boldsymbol{\eta}_{h}^{n}}{2}\right), \nabla \cdot \boldsymbol{v}_{h}^{s}\right\rangle_{\Omega_{0}^{s}}
$$

\section{$(2.16 \mathrm{~b})$}

$$
=\left\langle\boldsymbol{f}_{s}^{n+1}, \boldsymbol{v}_{h}^{s}\right\rangle_{\Omega_{0}^{s}}-\left\langle\mathcal{R}\left(\boldsymbol{u}_{h}^{n+1}, p_{h}^{n+1}\right), \mathcal{E}_{h}\left(\boldsymbol{v}_{h}^{s}\right)\right\rangle_{\Omega_{t^{n+1}}^{f}},
$$

\section{$(2.16 \mathrm{c})$}

$$
\left(\frac{\dot{\boldsymbol{\eta}}_{h}^{n+1}+\dot{\boldsymbol{\eta}}_{h}^{n}}{2}, \boldsymbol{v}_{h}^{s}\right)_{\Omega_{0}^{s}}=\left(\frac{\boldsymbol{\eta}_{h}^{n+1}-\boldsymbol{\eta}_{h}^{n}}{\delta t}, \boldsymbol{v}_{h}^{s}\right)_{\Omega_{0}^{s}}
$$

first equation with

$$
\mathcal{A}_{t^{n+1}}\left(\boldsymbol{x}_{0}\right)=\boldsymbol{x}_{0}+\operatorname{Ext}_{h}\left(\left.\widetilde{\boldsymbol{\eta}}_{h}^{n+1}\right|_{\Sigma_{0}}\right) .
$$

- Step 2: Solve the fluid-structure problem as in (2.16) but replace the momentum equation (2.16a) by the linearized version:

$$
\begin{aligned}
\rho_{f}\left(\left.\delta_{t} \boldsymbol{u}_{h}^{n+1}\right|_{\boldsymbol{x}_{0}}, \boldsymbol{v}_{h}^{f}\right)_{\Omega_{t^{n+1}}^{f}}+2 \mu\left(\boldsymbol{\epsilon}\left(\boldsymbol{u}_{h}^{n+1}\right), \boldsymbol{\epsilon}\left(\boldsymbol{v}_{h}^{f}\right)\right)_{\Omega_{t^{n+1}}^{f}} \\
+c_{h}\left(\widetilde{\boldsymbol{u}}_{h}^{n+1}-\boldsymbol{w}_{h}^{n+1}, \boldsymbol{u}_{h}^{n+1}, \boldsymbol{v}_{h}^{f}\right)_{\Omega_{t^{n+1}}^{f}}-\left(p_{h}^{n+1}, \nabla \cdot \boldsymbol{v}_{h}^{f}\right)_{\Omega_{t^{n+1}}^{f}} \\
+\left(\nabla \cdot \boldsymbol{u}_{h}^{n+1}, q_{h}\right)_{\Omega_{t^{n+1}}^{f}}=\left\langle\boldsymbol{f}_{f}^{n+1}, \boldsymbol{v}_{h}^{f}\right\rangle_{\Omega_{t^{n+1}}^{f}} .
\end{aligned}
$$

- Step 3: Check the stopping criterion. If it is not satisfied, update $\widetilde{\boldsymbol{\eta}}_{h}^{n+1}=$ $\boldsymbol{\eta}_{h}^{n+1}, \widetilde{\mathbf{u}}_{h}^{n+1}=\mathbf{u}_{h}^{n+1}$, and go to Step 1.

We have ended up with a fully discretized and linearized fluid-structure problem that can be solved by a linear solver. Notice that the fluid and structure problems are strongly coupled: The fluid solution depends on $\boldsymbol{\eta}_{h}^{n+1}$ through (2.16d), whereas to solve the structure problem in $(2.16 \mathrm{~b}), \mathbf{u}_{h}^{n+1}$ and $p_{h}^{n+1}$ are needed. Because of this 
implicit treatment of the coupling, the convergence of the fixed point algorithm described above is independent of the added-mass effect and therefore suitable for hemodynamics applications (see [2]).

2.3. The linear fluid-structure system. We aim at writing the fluid-structure system yielded by the linearized and fully discretized FSI problem. We start by writing the finite element approximation of the fluid unknowns:

$$
\begin{aligned}
\boldsymbol{u}_{h}^{n+1}\left(\boldsymbol{x}, t^{n+1}\right) & =\sum_{i \in \mathcal{N}_{f}} \boldsymbol{\phi}_{i}\left(\boldsymbol{x}, t^{n+1}\right)\left(\mathbf{U}_{f}^{n+1}\left(t^{n+1}\right)\right)_{i}+\sum_{j \in \mathcal{N}_{\sigma}} \boldsymbol{\phi}_{j}^{\sigma}\left(\boldsymbol{x}, t^{n+1}\right)\left(\mathbf{U}_{\sigma}^{n+1}\left(t^{n+1}\right)\right)_{j}, \\
2.20) \quad p_{h}^{n+1}(\boldsymbol{x}) & =\sum_{k \in \mathcal{N}_{p}} \pi_{k}\left(\boldsymbol{x}, t^{n+1}\right)\left(\mathbf{P}^{n+1}\left(t^{n+1}\right)\right)_{k},
\end{aligned}
$$

where $\mathbf{U}_{f}^{n+1}, \mathbf{U}_{\sigma}^{n+1}$, and $\mathbf{P}^{n+1}$ are the arrays of nodal values for the velocity of the inner nodes, the velocity of the interface nodes, and the pressure, respectively. Obviously, the finite element shape functions vary (in time) in the following way: $\phi_{i}\left(\boldsymbol{x}, t^{n}\right)=\mathcal{A}_{t^{n}}\left(\widehat{\phi}_{i}\left(\boldsymbol{x}_{0}\right)\right)$, where $\widehat{\boldsymbol{\phi}}_{i}\left(\boldsymbol{x}_{0}\right)$ are the Laplacian shape finite element functions on the reference grid $\hat{\mathcal{T}}_{h}(t)$.

We also set:
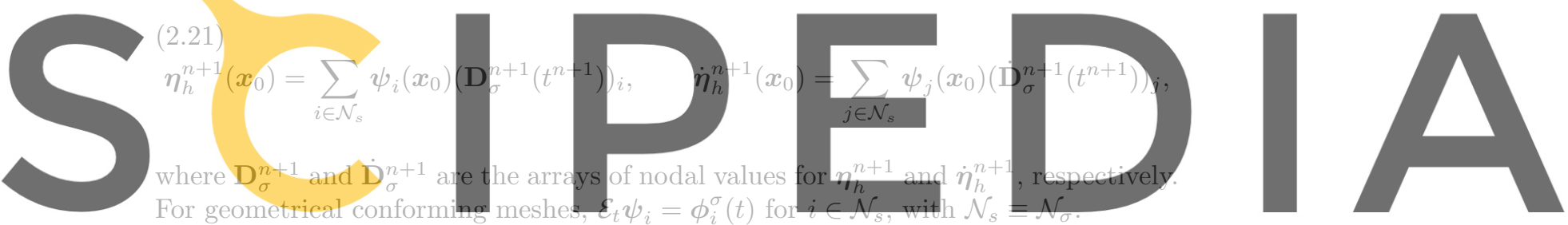

From (2.16d) we know that

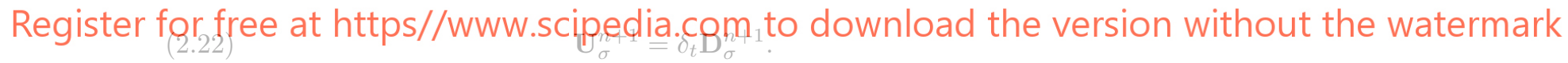

In order to write the fully discretized coupled problem for a given time value $t^{n+1}$, we need to define a set of matrices. Let us introduce the subindexes $\alpha$ and $\beta$ associated to the position of fluid nodes: The "value" $\sigma$ is used for nodes on $\Sigma, f$ otherwise. Superindexes $a$ and $b$ will denote nodes. Then

$$
\begin{array}{lll}
K_{\alpha \beta}^{a b}:=\nu\left(\nabla \phi_{a}, \nabla \phi_{b}\right)_{\Omega_{t^{n+1}}^{f}}+c_{h}\left(\widetilde{\boldsymbol{u}}_{h}^{n+1}-\boldsymbol{w}_{h}^{n+1}, \boldsymbol{\phi}_{a}, \boldsymbol{\phi}_{b}\right)_{\Omega_{t^{n+1}}^{f}}, & a \in \mathcal{N}_{\alpha}, \quad b \in \mathcal{N}_{\beta}, \\
M_{\alpha \beta}^{a b}:=\left(\phi_{a}, \phi_{b}\right)_{\Omega_{t^{n+1}}^{f}}, \quad C_{\alpha \beta}:=\frac{1}{\delta t} M_{\alpha \beta}+K_{\alpha \beta}, & a \in \mathcal{N}_{\alpha}, \quad b \in \mathcal{N}_{\beta}, \\
G_{\alpha}^{a b}:=-\left(\nabla \cdot \phi_{a}, \pi_{b}\right)_{\Omega_{t^{n+1}}^{f}}, \quad D_{\alpha}:=G_{\alpha}^{T}, & a \in \mathcal{N}_{\alpha}, \quad b \in \mathcal{N}_{p} .
\end{array}
$$

Let us denote with $N$ the matrix associated to the structure written in terms of $\mathbf{U}_{\sigma}^{n+1}$ by virtue of (2.22). Note that the fluid matrices are calculated over $\Omega_{t^{n+1}}^{f}$, which depends on the unknown $\boldsymbol{\eta}_{h}^{n+1}$.

At a given time value $t^{n+1},(2.18),(2.16 \mathrm{~b}),(2.16 \mathrm{c})$, and $(2.16 \mathrm{~d})$ can be written in matrix form as:

$$
A \mathbf{X}^{n+1}=\mathbf{b}^{n+1}
$$

Copyright $@$ by SIAM. Unauthorized reproduction of this article is prohibited. 
where

$$
A=\left[\begin{array}{ccc}
C_{f f} & G_{f} & C_{f \sigma} \\
D_{f} & 0 & D_{\sigma} \\
C_{\sigma f} & G_{\sigma} & C_{\sigma \sigma}+N
\end{array}\right], \quad \mathbf{X}^{n+1}=\left[\begin{array}{c}
\mathbf{U}_{f}^{n+1} \\
\mathbf{P}^{n+1} \\
\mathbf{U}_{\sigma}^{n+1}
\end{array}\right], \quad \mathbf{b}^{n+1}=\left[\begin{array}{c}
\mathbf{b}_{f}^{n+1} \\
\mathbf{0} \\
\mathbf{b}_{\sigma}^{n+1}
\end{array}\right] .
$$

The force term $\mathbf{b}_{f}^{n+1}$ accounts for body forces and time integration terms related to the fluid. The term $\mathbf{b}_{\sigma}^{n+1}$ accounts for the interface force due to the fluid, the structure terms related to body force, time integration, and the fact that the structure equation is stated in terms of $\mathbf{U}_{\sigma}^{n+1}$.

Remark 1. The algebraic splitting algorithms we introduce in this paper are based on the block structure of matrix $A$ in (2.24). Other time discretization schemes for the fluid and structure subproblems can be used without altering that structure. For example, by using a second order backward differentiation formula (BDF2) to discretize the fluid equations, only the coefficient multiplying the mass matrix in the C-matrices changes. Therefore, the procedures proposed in the next section can be easily extended to other time integration methods.

\section{Remark 2. For $d$-dimensional structures, system (2.24) becomes}

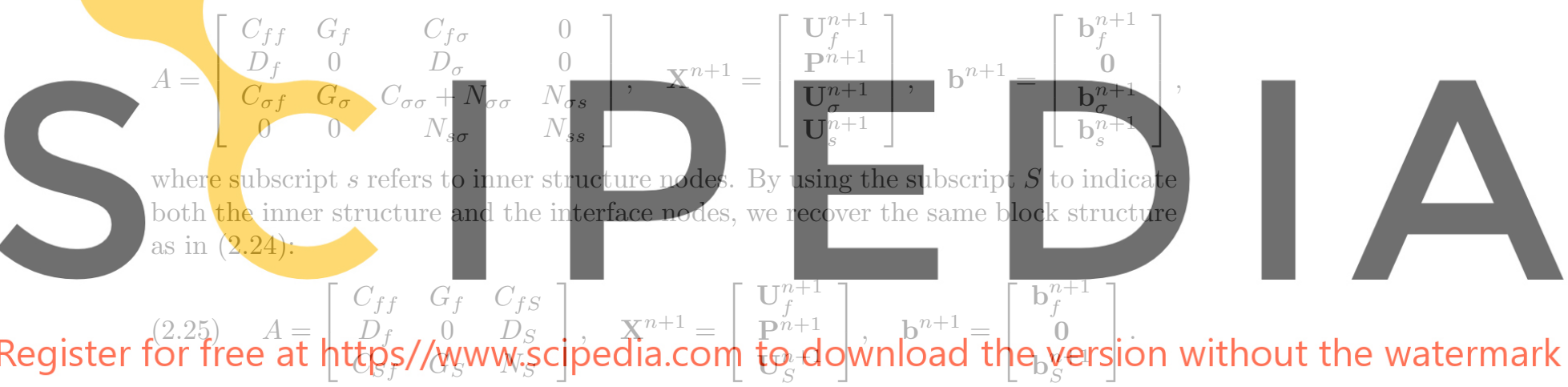

Thus, also the extension of our methodology to the case of $d$-dimensional structures is straightforward.

Remark 3. It is also possible to linearize the fluid and structure problems through Newton methods. Again, the block structure of matrix $A$ is left unchanged, and our procedures can be applied.

We do not consider the application of Newton methods to the FSI problem.

2.4. Block- $\boldsymbol{L} \boldsymbol{U}$ factorization of the coupled system. The solution of the fluid-structure system (2.23) by a monolithic method, such as a preconditioned Krylov method, can be very expensive. The associated matrix $A$ is indefinite, and for real applications its size is prohibitive. Therefore, we need to employ more efficient methods.

System (2.23) can be solved by using an exact block- $L U$ factorization of the fluidstructure system matrix $A$, for a suitable choice of the blocks to be decoupled. If the first block is that related to $\mathbf{U}_{f}^{n+1}$ and the second one is associated to the fluid pressure and structure velocity variables $\left[\mathbf{P}^{n+1}, \mathbf{U}_{\sigma}^{n+1}\right]$, the $L$ and $U$ factors read:

$$
A=\left[\begin{array}{ccc}
C_{f f} & 0 & 0 \\
D_{f} & S_{p p} & S_{p \sigma} \\
C_{\sigma f} & S_{\sigma p} & S_{\sigma \sigma}
\end{array}\right]\left[\begin{array}{ccc}
I & C_{f f}^{-1} G_{f} & C_{f f}^{-1} C_{f \sigma} \\
0 & I & 0 \\
0 & 0 & I
\end{array}\right]=: L U .
$$


The $S$-matrices are Schur complements. Their formal definition is

$$
\begin{array}{ll}
S_{p p}:=-D_{f} C_{f f}^{-1} G_{f}, & S_{p \sigma}:=D_{\sigma}-D_{f} C_{f f}^{-1} C_{f \sigma}, \\
S_{\sigma p}:=G_{\sigma}-C_{\sigma f} C_{f f}^{-1} G_{f}, & S_{\sigma \sigma}:=C_{\sigma \sigma}+N-C_{\sigma f} C_{f f}^{-1} C_{f \sigma} .
\end{array}
$$

These definitions involve the inverse fluid matrix $C_{f f}^{-1}$ (a dense matrix). The computational complexity of the exact $L U$ factorization can be reduced provided $C_{f f}^{-1}$ is approximated by a matrix cheaper to compute. This yields inexact factorizations which are still based on variable splitting but are much more computationally convenient than the exact splitting.

In the next sections, we consider different approximations of (2.26). They involve a perturbation error that can be reduced if the inexact factorization is carried out over the incremental system (instead of the nonincremental (2.23)):

\section{$A\left(\mathbf{X}^{n+1}-\mathbf{X}^{*}\right)=\mathbf{b}^{n+1}-A \mathbf{X}^{*}$,}

where $\mathbf{X}^{*}$ is the vector made of $\mathbf{U}_{f}^{*}, \mathbf{P}^{*}$, and $\mathbf{U}_{\sigma}^{*}$ which are predictions of $\mathbf{U}_{f}^{n+1}, \mathbf{P}^{n+1}$, and $\mathrm{U}_{\sigma}^{n+1}$, respectively. For instance, a first order prediction would be $\mathbf{X}^{*}=\mathbf{X}^{n}$.

3. Semi-implicit procedures for the FSI problem based on inexact factorization methods. A scheme for FST problems is said to be explicit if the fluid (or the structure) problem is conditions. Otherwise, have been proposed in to implicit methods with presence of a strong add The basic idea of a
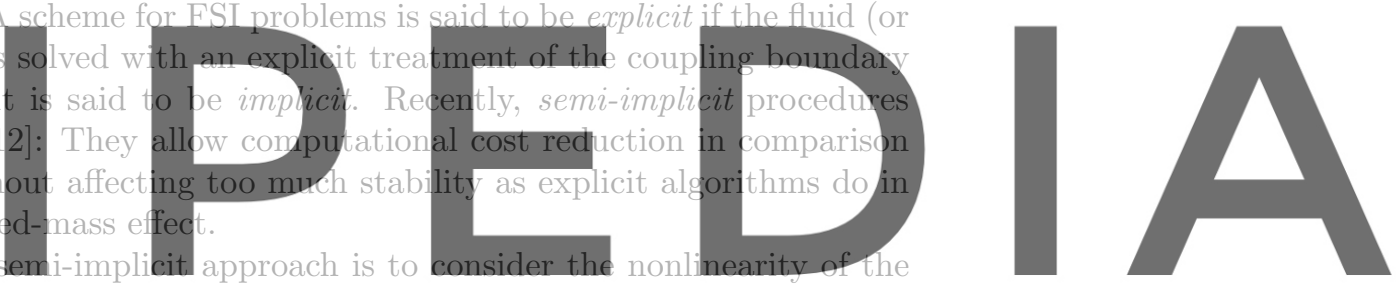

fluid-structure problem in an explicit way; i.e., only one iteration of the fixed point

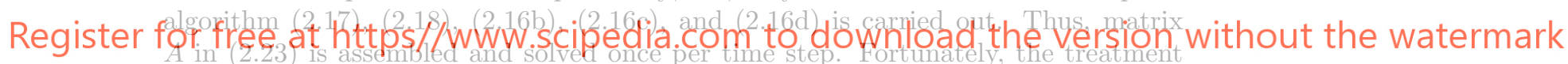

of the shape derivative in an explicit way does not affect seriously the stability of

the coupled problem, even when the added-mass effect is critical and fully explicit procedures are known to be unstable. In particular, if the FSI problem is discretized with a first order method (in time) and the condition

$$
\widetilde{\boldsymbol{u}}_{h}^{n+1}=\boldsymbol{w}^{n+1} \text { on } \Sigma_{t}
$$

is satisfied (e.g., by taking $\widetilde{\boldsymbol{u}}_{h}^{n+1}=\boldsymbol{u}_{h}^{n}$ and $\widetilde{\boldsymbol{\eta}}_{h}^{n+1}=\boldsymbol{\eta}_{h}^{n}$ ), the semi-implicit method keeps the stability properties of the implicit procedure (see [22]). In this case, we can avoid subiterating over the domain shape and the convective term without compromising stability.

Our goal is to derive semi-implicit algorithms from splitting techniques originally designed for the FSI problem at the fully discrete level instead of the differential one designed in [12]. The extension of algebraic splitting procedures to fluid-structure problems is not straightforward, especially when the added-mass effect is critical. We propose to adapt two methods to the coupled fluid-structure problem (2.23): the algebraic version of the Chorin-Temam method and the Yosida scheme. This will lead to two families of methods:

- pressure-interface correction (PIC) methods;

- fluid-structure Yosida (FSY) methods. 
PIC methods are presented for the first time, while FSY methods, already introduced in [23], are proposed in their incremental version. Differences and analogies between these procedures and the projection scheme in [12] will be analyzed in section 4 .

The third family of algorithms introduced in this section exploits the inexact factors of the PIC and FSY schemes as preconditioners, leading to predictor-corrector methods.

3.1. PIC methods. In this section, we consider an inexact factorization which is the FSI counterpart of the popular pressure-correction methods for pure fluid problems. We will call these methods PIC schemes (named after pressure-correction methods originally proposed by Chorin and Temam) since both the pressure and the interface velocity are treated explicitly (or ignored) in the first step and corrected in the second one.

The exact $L$ and $U$ factors in (2.26) are replaced by inexact ones in which $C_{f f}^{-1}$ is substituted by with the zeroth order term of its Neumann expansion:

$$
C_{f f}^{-1}=\left(\frac{1}{\delta t} M_{f f}+K_{f f}\right)^{-1}=\delta t M_{f f}^{-1}+\mathcal{O}\left(\delta t^{2}\right) \simeq \delta t M_{f f}^{-1} .
$$

Here $K_{f f}$ is the stiffness fluid matrix associated with the viscous term and the convective term. After approximating $C_{f f}^{-1}$ by $\delta t M_{f f}^{-1}$, the Schur complements matrices (2.27) become

$$
\begin{array}{ll}
S_{p p} \simeq T_{p p}:=-\delta t D_{f} M_{f f}^{-1} G_{f}, & S_{p \sigma} \simeq T_{p \sigma}:=D_{\sigma}-\delta t D_{f} M_{f f}^{-1} C_{f \sigma}, \\
S_{\sigma p} \simeq T_{\sigma p}:=G_{\sigma}-\delta t C_{\sigma f} M_{f f}^{-1} G_{f}, & S_{\sigma \sigma} \simeq T_{\sigma \sigma}:=C_{\sigma \sigma}+N-\delta t C_{\sigma f} M_{f f}^{-1} C_{f \sigma} .
\end{array}
$$

Consequently, the lower block-triangular matrix $L$ is approximated by:

$$
L_{P I C}:=\left[\begin{array}{ccc}
C_{f f} & 0 & 0 \\
D_{f} & T_{p p} & T_{p \sigma} \\
C_{\sigma f} & T_{\sigma p} & T_{\sigma \sigma}
\end{array}\right]
$$

By using the same approximation (3.1) for the upper block-triangular matrix $U$ (2.26), the following inexact $U$ factor is obtained:

$$
U_{P I C}:=\left[\begin{array}{ccc}
I & \delta t M_{f f}^{-1} G_{f} & \delta t M_{f f}^{-1} C_{f \sigma} \\
0 & I & 0 \\
0 & 0 & I
\end{array}\right]
$$

The system matrix for the PIC scheme is obtained by replacing matrices $L$ and $U$ with $L_{P I C}$ and $U_{P I C}$ :

$$
A_{P I C}=L_{P I C} U_{P I C}=\left[\begin{array}{ccc}
C_{f f} & \delta t C_{f f} M_{f f}^{-1} G_{f} & \delta t C_{f f} M_{f f}^{-1} C_{f \sigma} \\
D_{f} & 0 & D_{\sigma} \\
C_{\sigma f} & G_{\sigma} & C_{\sigma \sigma}+N
\end{array}\right] .
$$

Let us apply this inexact factorization to the incremental version of the FSI system (2.28) (the nonincremental version is nothing but a special case corresponding to the trivial choice $\mathbf{X}^{*}=\mathbf{0}$ ). We rearrange the resulting system into three uncoupled steps: 
1. computation of the intermediate velocity:

$$
C_{f f} \widetilde{\mathbf{U}}_{f}^{n+1}=\mathbf{b}_{f}^{n+1}-G_{f} \mathbf{P}^{*}-C_{f \sigma} \mathbf{U}_{\sigma}^{*} ;
$$

2. solution of the coupled pressure-interface system:

$$
\begin{aligned}
& {\left[\begin{array}{ll}
T_{p p} & T_{p \sigma} \\
T_{\sigma p} & T_{\sigma \sigma}
\end{array}\right]\left[\begin{array}{c}
\mathbf{P}^{n+1}-\mathbf{P}^{*} \\
\mathbf{U}_{\sigma}^{n+1}-\mathbf{U}_{\sigma}^{*}
\end{array}\right]} \\
& (3.4 \mathrm{~b}) \quad=\left[\begin{array}{c}
-D_{f} \widetilde{\mathbf{U}}_{f}^{n+1} \\
\mathbf{b}_{\sigma}^{n+1}-C_{\sigma f} \widetilde{\mathbf{U}}_{f}^{n+1}
\end{array}\right]-\left[\begin{array}{cc}
0 & D_{\sigma} \\
G_{\sigma} & C_{\sigma \sigma}+N
\end{array}\right]\left[\begin{array}{c}
\mathbf{P}^{*} \\
\mathbf{U}_{\sigma}^{*}
\end{array}\right] ;
\end{aligned}
$$

3. computation of the end-of-step velocity:

$$
\frac{1}{\delta t} M_{f f} \mathbf{U}_{f}^{n+1}=\frac{1}{\delta t} M_{f f} \tilde{\mathbf{U}}_{f}^{n+1}-G_{f}\left(\mathbf{P}^{n+1}-\mathbf{P}^{*}\right)-C_{f \sigma}\left(\mathbf{U}_{\sigma}^{n+1}-\mathbf{U}_{\sigma}^{*}\right)
$$

Note that the prediction of the fluid velocity $\mathbf{U}_{f}^{*}$ does not enter in the PIC scheme (3.4); therefore it cannot affect the order of accuracy in time of the method. The perturbation terms due to the inexact factors $L_{P I C}$ and $U_{P I C}$ (see section 5) depend only on $\mathbf{P}^{*}$ and $\mathbf{U}_{\sigma}^{*}$. Different approximations for $\mathbf{P}^{*}$ and $\mathbf{U}_{\sigma}^{*}$ can be considered:

$$
\begin{array}{ll}
\mathbf{P}^{*}=\mathbf{0}, \mathbf{U}_{\sigma}^{*}=\mathbf{0}, & \text { 0th order approximation, } \\
\mathbf{P}^{*}=\mathbf{P}^{n}, \mathbf{U}_{\sigma}^{*}=\mathbf{U}_{\sigma}^{n}, & \text { 1st order approximation, } \\
\mathbf{P}^{*}=2 \mathbf{P}^{n}-\mathbf{P}^{n-1}, \mathbf{U}_{\sigma}^{*}=2 \mathbf{U}_{\sigma}^{n}-\mathbf{U}_{\sigma}^{n-1}, & \text { 2nd order approximation. }
\end{array}
$$

With PIC schemes, we pass from an indefinite system coupling velocity components, pressure, and structure unknowns to a set of smaller systems. For instance, by neglecting convective terms, the PIC schemes involve the solution of the following:

1. A definite system (3.4a) for the fluid velocity. In case we use the Laplace form of the viscous term, every velocity component is decoupled from one another.

2. A definite system (3.4b) coupling the fluid and the structure through the coupling of pressure and interface velocity. Therefore, with PIC schemes, the dimension of the fluid-structure system affected by the added-mass effect has been clearly reduced. In the following we denote by $T$ the system matrix of the pressure-interface problem. Further comments on how to solve system (3.4b) are made in section 6 .

3. A cheap system (3.4c) with a diagonal system matrix if we apply mass lumping.

In conclusion, this method not only reduces the dimension of the fluid-structure system but changes its nature, too, becoming much more convenient from a computational point of view.

Remark 4. It is known that pressure-correction methods for the fluid problem introduce an artificial boundary condition over the pressure on Dirichlet (velocity) boundaries. At the discrete level, $\partial p / \partial \boldsymbol{n}=0$ is imposed in a weak form on these boundaries. With the PIC scheme, a more consistent boundary condition is imposed over the interface, due to the presence of the interface velocity terms (see section 4).

Remark 5. The computational efficiency of the approximation (3.1) is evident if we replace the original mass matrix with a suitable diagonal matrix obtained by quadrature formulas for the space integrals (the so-called mass lumping; see [27]). In any case, we understand that $M_{f f}^{-1}$ is "easy" to compute. 
Remark 6. In the case of $d$-dimensional structures, the inexact block- $L U$ factorization is carried out on matrix $A$ in (2.25). The first block is still associated to $\mathbf{U}_{f}^{n+1}$, while the second one is related to the variables $\left[\mathbf{P}^{n+1}, \mathbf{U}_{S}^{n+1}\right]$. The resulting PIC scheme is obtained by replacing in (3.4) subindex $\sigma$ with $S$.

3.2. FSY methods. The Yosida method for fluid problems was introduced in $[32,26]$ as an inexact factorization of the system matrix arising from the numerical approximation of the Navier-Stokes equations for incompressible flows. Here we extend it to the FSI problem (2.23).

The inexact factorization of matrix $A(2.24)$ is again based on the approximation (3.1), but it is used only on the lower block-triangular matrix, i.e., for the evaluation of the Schur complements. In the $U$ factor, matrix $C_{f f}^{-1}$ is not approximated. Thus, the inexact factorization we use in this case is simply

$$
A_{F S Y}=L_{P I C} U \text {. }
$$

The incremental version of the FSY scheme can be accomplished in three steps: The first two steps coincide with (3.4a) and (3.4b), whereas the third one becomes

- computation of the end-of-step velocity:

$$
C_{f f} \mathbf{U}_{f}^{n+1}=C_{f f} \widetilde{\mathbf{U}}_{f}^{n+1}-G_{f}\left(\mathbf{P}^{n+1}-\mathbf{P}^{*}\right)-C_{f \sigma}\left(\mathbf{U}_{\sigma}^{n+1}-\mathbf{U}_{\sigma}^{*}\right) .
$$

The latter step differs from (3.4c) and is actually more expensive due to the presence of the stiffness matrix $C_{f f}$.

3.3. Predictor-corrector methods. The nonincremental version of pressurecorrection methods has led to new iterative algorithms for the solution of the monolithic fluid system (see [7]). In this section, we suggest to use $A_{P I C}$ and $A_{F S Y}$ as preconditioners, together with Richardson iterations; however, more efficient iterative procedures, such as GMRES, could be considered. By using, e.g., $A_{P I C}$ as a preconditioner, we have to solve: Given $\mathbf{X}^{n+1, k}$, find $\mathbf{X}^{n+1, k+1}$ such that

$$
A_{P I C} \delta \mathbf{X}^{n+1, k+1}=\mathbf{b}^{n+1}-A \mathbf{X}^{n+1, k}, \quad k \geq 0,
$$

until convergence. The operator $\delta x^{n+1, k+1}=x^{n+1, k+1}-x^{n+1, k}$ denotes the backward increment of iteration $k+1$. We can also write this scheme in the fashion of (3.4). In this case, iteration $k+1$ of the predictor-corrector scheme consists of solving three different steps:

1. computation of the intermediate velocity:

$$
C_{f f} \widetilde{\mathbf{U}}_{f}^{n+1, k+1}=\mathbf{b}_{f}^{n+1}-G_{f} \mathbf{P}^{n+1, k}-C_{f \sigma} \mathbf{U}_{\sigma}^{n+1, k} ;
$$

2. solution of the pressure-interface system:

$$
\begin{aligned}
& {\left[\begin{array}{cc}
T_{p p} & T_{p \sigma} \\
T_{\sigma p} & T_{\sigma \sigma}
\end{array}\right]\left[\begin{array}{l}
\delta \mathbf{P}^{n+1, k+1} \\
\delta \mathbf{U}_{\sigma}^{n+1, k+1}
\end{array}\right]} \\
& (3.7 \mathrm{~b})=\left[\begin{array}{c}
-D_{f} \widetilde{\mathbf{U}}_{f}^{n+1, k+1} \\
\mathbf{b}_{\sigma}^{n+1}-C_{\sigma f} \widetilde{\mathbf{U}}_{f}^{n+1, k+1}
\end{array}\right]-\left[\begin{array}{cc}
0 & D_{\sigma} \\
G_{\sigma} & C_{\sigma \sigma}+N
\end{array}\right]\left[\begin{array}{c}
\mathbf{P}^{n+1, k} \\
\mathbf{U}_{\sigma}^{n+1, k}
\end{array}\right] ;
\end{aligned}
$$

3. computation of the end-of-step velocity:

$$
\frac{1}{\delta t} M_{f f} \mathbf{U}_{f}^{n+1, k+1}=\frac{1}{\delta t} M_{f f} \widetilde{\mathbf{U}}_{f}^{n+1, k+1}-G_{f} \delta \mathbf{P}^{n+1, k+1}-C_{f \sigma} \delta \mathbf{U}_{\sigma}^{n+1, k+1} .
$$


Similarly, by taking $A_{F S Y}$ as a preconditioner we get a different version of the predictor-corrector method, which shares steps 1 and 2 with (3.7) but replaces (3.7c) with:

$$
C_{f f} \mathbf{U}_{f}^{n+1, k+1}=C_{f f} \widetilde{\mathbf{U}}_{f}^{n+1, k+1}-G_{f} \delta \mathbf{P}^{n+1, k}-C_{f \sigma} \delta \mathbf{U}_{\sigma}^{n+1, k} .
$$

In a compact form, the predictor-corrector method based on the FSY algorithm reads: Given $\mathbf{X}^{n+1, k}$, solve

$$
A_{F S Y} \delta \mathbf{X}^{n+1, k+1}=\mathbf{b}^{n+1}-A \mathbf{X}^{n+1, k}
$$

until convergence.

We can now see that the end-of-step velocity does not play any role in the iterative process, because $\mathbf{U}_{f}^{n+1, k}$ does not appear in the iteration $k+1$. Therefore, (3.7c) (or (3.8) for FSY) can be disregarded without perturbing the convergence of the algorithm. The resulting predictor-corrector method reduces to (3.7a) and (3.7b) upon replacing $\widetilde{\mathbf{U}}_{f}^{n+1, k+1}$ with $\mathbf{U}_{f}^{n+1, k+1}$. This is a preconditioned Richardson iteration with preconditioner $L_{P I C}$; that is,

$$
L_{P I C} \delta \mathbf{X}^{n+1, k+1}=\mathbf{b}^{n+1}-A \mathbf{X}^{n+1, k} .
$$

We remind the reader that $L_{P I C}=L_{F S Y}$.

The convergence of the predictor-corrector method is added-mass-independent. Fluid and structure are not fully decoupled, and we treat the added-mass effect implicitly. This is essential for the good convergence properties of the predictor-corrector iterative procedure when dealing with hemodynamics problems (see section 7).

Predictor-corrector methods are particularly well suited when considering domain and/or convective terms in an implicit way. In this case, the FSI system has to be evaluated as many times as implicit iterations. Therefore, we can consider one-loop algorithms, i.e., dealing with implicit treatment and predictor-corrector iterations with only one external loop. One-loop algorithms were designed in [1] for aeroelastic applications. Therein, the predictor-corrector method dealt with the added-mass effect, because fluid and structure problems were fully decoupled (the main difference with respect to the one suggested in this work).

Remark 7. The preconditioners suggested in this section are based on approximation (3.1) for the Schur complements. Improved preconditioners, approximating also the effect of the convective and diffusive terms, have been successfully used for the numerical approximation of the Navier-Stokes equations (see $[31,11,18]$ ). The extension of these preconditioners to the FSI system is an object of research by the authors.

Remark 8. From the FSI system (2.24) we can write the pressure-interface (or pressure-structure, for $d$-dimensional structures) system:

$$
\left[\begin{array}{cc}
S_{p p} & S_{p \sigma} \\
S_{\sigma p} & S_{\sigma \sigma}
\end{array}\right]\left[\begin{array}{c}
\mathbf{P}^{n+1} \\
\mathbf{U}_{\sigma}^{n+1}
\end{array}\right]=\left[\begin{array}{c}
-D_{f} C_{f f}^{-1} \mathbf{b}_{f}^{n+1} \\
\mathbf{b}_{\sigma}^{n+1}-C_{\sigma f} C_{f f}^{-1} \mathbf{b}_{f}^{n+1}
\end{array}\right]
$$

that is obtained upon formal elimination of the flow velocity $\mathbf{U}_{f}^{n+1}$. Our predictorcorrector method can be interpreted as a preconditioned Richardson iterative solver on (3.11) with preconditioner

$$
\left[\begin{array}{ll}
T_{p p} & T_{p \sigma} \\
T_{\sigma p} & T_{\sigma \sigma}
\end{array}\right] .
$$


Needless to say, this pressure-structure Schur complement (3.12) can also serve as a preconditioner for Krylov iterative solvers, such as GMRES.

Note that a single Richardson iteration for system (3.11) with preconditioner (3.12) differs from a Dirichlet-Neumann iteration applied directly on the original FSI system (2.24).

4. Comparison between inexact factorization-based methods and the projection scheme. In this section, we compare the projection-based coupling scheme in [12] with the semi-implicit procedures introduced in this paper (section $3)$.

In [12], the Chorin-Temam method applied to the fluid problem leads to an algorithm that obtains the intermediate velocity by using a prediction of the structure displacement, and then, at a second step, it solves the end-of-step velocity and pressure coupled to the structure model. Furthermore, the load exerted by the fluid on the structure is computed in a peculiar residual way: The diffusive and convective terms of this fluid residual are evaluated by using the intermediate velocity, whereas the time derivative by using the end-of-step velocity. Since the fluid problem has been split at the continuous level (in space), only the normal component of the velocity can be imposed.

This approach could also be considered at the fully discrete level by using a pressure-correction method for the fluid problem obtained as an inexact factorization of the fluid matrix (see [24]). The main advantage of this approach is the fact that boundary conditions are accounted for intrinsically, allowing, e.g., one to hold the continuity of velocities over the boundary. The discrete counterpart of the method suggested in [12] reads as:

- Step 1: Intermediate velocity.

$$
C_{f f} \widetilde{\mathbf{U}}_{f}^{n+1}=\mathbf{b}_{f}^{n+1}-C_{f \sigma} \widetilde{\mathbf{U}}_{\sigma}^{n+1} .
$$

- Step 2: End-of-step velocity, pressure, and interface-velocity.

$$
\begin{aligned}
& {\left[\begin{array}{ccc}
\frac{1}{\delta t} M_{f f} & G_{f} & \frac{1}{\delta t} M_{f \sigma} \\
D_{f} & 0 & D_{\sigma} \\
\frac{1}{\delta t} M_{\sigma f} & G_{\sigma} & \frac{1}{\delta t} M_{\sigma \sigma}+N
\end{array}\right]\left[\begin{array}{c}
\mathbf{U}_{f}^{n+1} \\
\mathbf{P}^{n+1} \\
\mathbf{U}_{\sigma}^{n+1}
\end{array}\right]} \\
& =\left[\begin{array}{c}
\frac{1}{\delta t} M_{f f} \widetilde{\mathbf{U}}_{f}^{n+1}+\frac{1}{\delta t} M_{f \sigma} \widetilde{\mathbf{U}}_{\sigma}^{n+1} \\
\mathbf{0} \\
\mathbf{b}_{\sigma}^{n+1}-K_{\sigma f} \widetilde{\mathbf{U}}_{f}^{n+1}-K_{\sigma \sigma} \widetilde{\mathbf{U}}_{\sigma}^{n+1}
\end{array}\right],
\end{aligned}
$$

where $\widetilde{\mathbf{U}}_{\sigma}^{n+1}$ is computed by means of a second order extrapolation for the interface displacement, calculated at a step 0 . In the second step, the diffusive and convective terms are treated explicitly, even for the interface velocity, and fluid velocity, pressure, and structure unknowns are coupled.

The scheme (4.1) cannot be derived from an inexact factorization of the FSI system matrix in (2.23). In order to compare the discrete counterpart of the projection method in [12] with PIC and FSY schemes, we need to reformulate the second step and rearrange (4.1) in a three-step scheme. Through the Schur complements of the system matrix in (4.1b) it is now possible to decouple the computation of $\mathbf{U}_{f}^{n+1}$ from Step 2. Then after the first step (4.1a), Step 2 of the algorithm becomes as follows:

- Step 2: Solution of the coupled pressure-interface system.

$$
\left[\begin{array}{cc}
T_{p p} & \hat{T}_{p \sigma} \\
\hat{T}_{\sigma p} & \hat{T}_{\sigma \sigma}
\end{array}\right]\left[\begin{array}{c}
\mathbf{P}^{n+1} \\
\mathbf{U}_{\sigma}^{n+1}
\end{array}\right]=\left[\begin{array}{c}
-D_{f} \widetilde{\mathbf{U}}_{f}^{n+1}-D_{f} M_{f f}^{-1} M_{f \sigma} \widetilde{\mathbf{U}}_{\sigma}^{n+1} \\
\mathbf{b}_{\sigma}^{n+1}-K_{\sigma f} \widetilde{\mathbf{U}}_{f}^{n+1}-K_{\sigma \sigma} \widetilde{\mathbf{U}}_{\sigma}^{n+1}
\end{array}\right]
$$


- Step 3: Computation of the end-of-step velocity.

$$
\frac{1}{\delta t} M_{f f} \mathbf{U}_{f}^{n+1}=\frac{1}{\delta t} M_{f f} \widetilde{\mathbf{U}}_{f}^{n+1}-G_{f} \mathbf{P}^{n+1}-\frac{1}{\delta t} M_{f \sigma}\left(\mathbf{U}_{\sigma}^{n+1}-\widetilde{\mathbf{U}}_{\sigma}^{n+1}\right) .
$$

Matrices $\hat{T}_{p \sigma}, \hat{T}_{\sigma p}$, and $\hat{T}_{\sigma \sigma}$ are further approximations of the approximated Schur complements (3.2), obtained by replacing $C_{\alpha \beta}$ with $\frac{1}{\delta t} M_{\alpha \beta}$ (see section 2.3):

$$
\begin{aligned}
& \hat{T}_{p \sigma}:=D_{\sigma}-D_{f} M_{f f}^{-1} M_{f \sigma}, \quad \hat{T}_{\sigma p}:=G_{\sigma}-M_{\sigma f} M_{f f}^{-1} G_{f}, \\
& \hat{T}_{\sigma \sigma}:=\frac{1}{\delta t} M_{\sigma \sigma}+N-\frac{1}{\delta t} M_{\sigma f} M_{f f}^{-1} M_{f \sigma} .
\end{aligned}
$$

The algebraic counterpart of the semi-implicit projection algorithm in [12] shares Step 1 with the other two methods (with zeroth order approximation for the pressure and a different first order approximation for the interface velocity), and like them at the second step it couples only the pressure term to the structure. Nonetheless, $\mathbf{P}^{n+1}$ and $\mathbf{U}_{\sigma}^{n+1}$ are computed through a different, simplified, system. Also Step 3 is simplified with respect to (3.4c): Matrix $C_{f \sigma}$ is replaced by $\frac{1}{\delta t} M_{f \sigma}$.

In particular, we remark the differences between the PIC method and algorithm (4.1a), (4.2a), and (4.2b). The PIC scheme extends the inexact factorization of the algebraic Chorin-Temam method to the FSI system, while algorithm (4.1a), (4.2a), and $(4.2 \mathrm{~b})$ derives from the discretization of the differential Chorin-Temam method applied to the fluid only. For the latter, the coupling with the structure is given by the boundary condition at the second step of the Chorin-Temam scheme, whose differential form is

$$
\begin{array}{ll}
\frac{1}{\delta t}\left(\boldsymbol{u}^{n+1}-\widetilde{\boldsymbol{u}}^{n+1}\right)+\nabla p^{n+1}=0 & \text { in } \Omega_{t^{n+1}}^{f}, \\
\nabla \cdot \boldsymbol{u}^{n+1}=0 & \text { in } \Omega_{t^{n+1}}^{f},
\end{array}
$$

where these velocities over the interface hold:

$$
\begin{aligned}
& \widetilde{\boldsymbol{u}}^{n+1}=\widetilde{\boldsymbol{u}}_{\sigma}^{n+1}, \\
& \boldsymbol{u}^{n+1} \cdot \boldsymbol{n}_{f}=\boldsymbol{u}_{\sigma}^{n+1} \cdot \boldsymbol{n}_{f} .
\end{aligned}
$$

We indicate with $\boldsymbol{u}_{\sigma}^{n+1}$ and $\widetilde{\boldsymbol{u}}_{\sigma}^{n+1}$ the interface velocity and its prediction calculated at a step 0 of the scheme, respectively. By multiplying (4.3a) evaluated on $\Sigma_{t^{n+1}}$ by $\boldsymbol{n}_{f}$, we obtain the boundary condition imposed over the pressure on the Dirichlet boundaries:

$$
\frac{\partial p^{n+1}}{\partial \boldsymbol{n}_{f}}=-\frac{1}{\delta t}\left(\boldsymbol{u}_{\sigma}^{n+1}-\widetilde{\boldsymbol{u}}_{\sigma}^{n+1}\right) \cdot \boldsymbol{n}_{f}
$$

The same boundary condition is imposed in a weak form by the PIC scheme.

The matrix $D_{f} M_{f f}^{-1} G_{f}=-\frac{1}{\delta t} T_{p p}$ is sometimes referred to as discrete Laplacian, because of the analogies with the discretization of the Laplace operator $-\triangle=-\nabla$. $(\nabla)$. Another way to discretize the scheme proposed in [12] would be to replace $D_{f} M_{f f}^{-1} G_{f}$ at Step 2 (4.2a) with the classical discretization of the Laplace operator.

4.1. Variations on the semi-implicit projection scheme. Let us consider some slight modifications of algorithm (4.1). First of all, we can handle the pressure term by using the incremental version of the Chorin-Temam method for the fluid 
problem (first order approximation) in order to make the scheme less dissipative and improve the accuracy. Then we can manipulate (4.1b) in order to decouple the computation of the end-of-step velocity from the pressure and structure unknowns. After rewriting it as

$$
\begin{aligned}
& -\delta t D_{f} M_{f f}^{-1} G_{f}\left(\mathbf{P}^{n+1}-\mathbf{P}^{n}\right)+\left(D_{\sigma}-D_{f} M_{f f}^{-1} M_{f \sigma}\right) \\
& \quad=-D_{f} \widetilde{\mathbf{U}}_{f}^{n+1}-D_{f} M_{f f}^{-1} M_{f \sigma} \widetilde{\mathbf{U}}_{\sigma}^{n+1}, \\
& \begin{array}{r}
\frac{1}{\delta t} M_{\sigma f} \mathbf{U}_{f}^{n+1}+G_{\sigma} \mathbf{P}^{n+1}+\left(\frac{1}{\delta t} M_{\sigma \sigma}+N\right) \mathbf{U}_{\sigma}^{n+1}=\mathbf{b}_{\sigma}^{n+1}-K_{\sigma f} \widetilde{\mathbf{U}}_{f}^{n+1}-K_{\sigma \sigma} \widetilde{\mathbf{U}}_{\sigma}^{n+1},
\end{array}
\end{aligned}
$$

we suggest to evaluate the stress of the fluid on the structure with the intermediate velocity. The resulting algorithm reads as follows:

1. intermediate velocity:

$$
C_{f f} \widetilde{\mathbf{U}}_{f}^{n+1}=\mathbf{b}_{f}^{n+1}-G_{f} \mathbf{P}^{n}-C_{f \sigma} \widetilde{\mathbf{U}}_{\sigma}^{n+1} ;
$$

2. pressure-interface problem:

$$
\begin{array}{r}
-\delta t D_{f} M_{f f}^{-1} G_{f}\left(\mathbf{P}^{n+1}-\mathbf{P}^{n}\right)+\left(D_{\sigma}-D_{f} M_{f f}^{-1} M_{f \sigma}\right) \mathbf{U}_{\sigma}^{n+1} \\
=-D_{f} \widetilde{\mathbf{U}}_{f}^{n+1}-D_{f} M_{f f}^{-1} M_{f \sigma} \widetilde{\mathbf{U}}_{\sigma}^{n+1} \\
G_{\sigma} \mathbf{P}^{n+1}+N \mathbf{U}_{\sigma}^{n+1}=\mathbf{b}_{\sigma}^{n+1}-C_{\sigma f} \widetilde{\mathbf{U}}_{f}^{n+1}-C_{\sigma \sigma} \widetilde{\mathbf{U}}_{\sigma}^{n+1}
\end{array}
$$

3. end-of-step velocity:

(4.6c)

$$
\frac{1}{\delta t} M_{f f} \mathbf{U}_{f}^{n+1}=\frac{1}{\delta t} M_{f f} \widetilde{\mathbf{U}}_{f}^{n+1}-G_{f}\left(\mathbf{P}^{n+1}-\mathbf{P}^{n}\right)-\frac{1}{\delta t} M_{f \sigma}\left(\mathbf{U}_{\sigma}^{n+1}-\widetilde{\mathbf{U}}_{\sigma}^{n+1}\right) .
$$

The advantage of this new scheme with respect to (4.1a), (4.2a), and (4.2b) is that the second equation of Step 2 involves no Schur complement and is therefore easier (and cheaper) to solve. Again, matrix $D_{f} M_{f f}^{-1} G_{f}$ in the first equation of (4.6b) might be replaced by the classical discretization of the Laplace operator, further simplifying Step 2.

Method (4.6) couples implicitly only the pressure term, while the rest of the fluid load is treated explicitly. Then it is expected to be well-suited for problems where the effect of the diffusive stress exerted by the fluid on the structure is less important than the one of the pressure.

These modifications could also be considered at the continuous level. Instead of (4.6b), we would get the pressure from the classical pressure Poisson equation with boundary condition (4.5).

These variations on the semi-implicit scheme in [12] are proposed because of their simplicity and good properties, but no numerical results on them are shown in this work.

5. Analysis of the perturbation error. At every time step, the inexact factorization of the system matrix $A$ by either PIC or FSY schemes perturbs the FSI system. The solution of a semi-implicit monolithic algorithm, which solves system (2.28) by, e.g., a global preconditioned GMRES, differs from the solutions of the PIC and FSY methods. This section is devoted to the identification of the perturbation terms introduced by the two schemes in order to infer the (formal) order of accuracy of the method in time. The results are confirmed by the numerical experiments in section 7.2 . 


\subsection{Perturbation terms for PIC schemes. By setting}

$$
A_{P I C}=A+E_{P I C}
$$

and subtracting $A$ from $A_{P I C}(3.3)$, we can calculate the perturbation matrix $E_{P I C}$ :

$$
E_{P I C}=\delta t\left[\begin{array}{ccc}
0 & K_{f f} M_{f f}^{-1} G_{f} & K_{f f} M_{f f}^{-1} C_{f \sigma} \\
0 & 0 & 0 \\
0 & 0 & 0
\end{array}\right] .
$$

The PIC scheme perturbs only the momentum conservation equation for the fluid but not the mass conservation equation nor the structure equation. Mass conservation is an excellent feature when considering problems with free surfaces or structures over fluid boundaries.

The incremental PIC scheme can be written as a monolithic system with a perturbed momentum equation:

$$
C_{f f} \widetilde{\mathbf{U}}_{f}^{n+1}+G_{f} \mathbf{P}^{*}+C_{f \sigma} \mathbf{U}_{\sigma}^{*}=\mathbf{b}_{f}^{n+1}+\mathbf{e}_{P I C},
$$

with

$$
\begin{aligned}
\mathbf{e}_{P I C}= & -\delta t K_{f f} M_{f f}^{-1} G_{f}\left(\mathbf{P}^{n+1}-\mathbf{P}^{*}\right)-\delta t K_{f f} M_{f f}^{-1} C_{f \sigma}\left(\mathbf{U}_{\sigma}^{n+1}-\mathbf{U}_{\sigma}^{*}\right) \\
= & -\delta t K_{f f} M_{f f}^{-1} G_{f}\left(\mathbf{P}^{n+1}-\mathbf{P}^{*}\right)-K_{f f} M_{f f}^{-1} M_{f \sigma}\left(\mathbf{U}_{\sigma}^{n+1}-\mathbf{U}_{\sigma}^{*}\right) \\
& +\delta t K_{f f} M_{f f}^{-1} K_{f \sigma}\left(\mathbf{U}_{\sigma}^{n+1}-\mathbf{U}_{\sigma}^{*}\right) .
\end{aligned}
$$

We have identified three different perturbation terms, one related to the pressure and two related to the interface velocity. Should $\mathbf{P}^{*}$ be a $q_{p}$ th order approximation of $\mathbf{P}^{n+1}$, the pressure term is of order $\mathcal{O}\left(\delta t^{q_{p}+1}\right)$. With regard to interface velocity terms, the one related to $K_{f \sigma}$ is of order $\mathcal{O}\left(\delta t^{q_{\sigma}+1}\right), q_{\sigma}$ being the order of approximation of $\mathbf{U}_{\sigma}^{*}$. However, we lose one order of accuracy in the term related to $M_{f \sigma}$. Therefore, in order to get a first order PIC scheme, it is enough to take $\mathbf{P}^{*}=\mathbf{0}$ and $\mathbf{U}_{\sigma}^{*}=\mathbf{U}_{\sigma}^{n}$. Anyway, it is advised to use a first order pressure approximation $\mathbf{P}^{*}=\mathbf{P}^{n}$ for reducing drastically the numerical dissipation without increasing CPU cost. In this case, the splitting error related to the pressure is second order in time.

We choose therefore $\mathbf{U}_{\sigma}^{*}=\mathbf{U}_{\sigma}^{n}$ in (3.4) in order to get the desired accuracy and $\mathbf{P}^{*}=\mathbf{P}^{n}$ in order to reduce the numerical dissipation. Also for the fluid problem alone numerical experiments show that a zeroth order approximation for the pressure leads to splitting procedures that, even though first order in time, are plagued by severe numerical dissipation (see [6]). The numerical results that are shown in section 7 are obtained with this incremental PIC scheme.

5.2. Perturbation terms for FSY schemes. A theoretical analysis of Yosida schemes for the numerical approximation of the Navier-Stokes equations has been carried out in [25]. Therein, strong stability results and optimal error estimates are proved. The matrix

$$
Y=\delta t^{-1} C_{f f}^{-1}-M_{f f}^{-1}=\mathcal{O}(\delta t),
$$

discussed in [25], plays an important role in the analysis of FSY methods. By setting

$$
A_{F S Y}=A+E_{F S Y},
$$

Copyright $@$ by SIAM. Unauthorized reproduction of this article is prohibited. 
performing the matrix-product in (3.5), and subtracting $A$ from it, we obtain the following expression for the perturbation matrix:

$$
E_{F S Y}=\delta t\left[\begin{array}{ccc}
0 & 0 & 0 \\
0 & D_{f} Y G_{f} & D_{f} Y C_{f \sigma} \\
0 & C_{\sigma f} Y G_{\sigma} & C_{\sigma f} Y C_{f \sigma}
\end{array}\right] .
$$

Note that this time the perturbation affects both the mass conservation and the structure equations, while PIC schemes perturb only the momentum conservation equation for the fluid. To identify the order of the perturbation errors we can write the FSY problem as a perturbed monolithic system. The perturbed mass conservation equation is

$$
D_{f} \mathbf{U}_{f}^{n+1}+D_{\sigma} \mathbf{U}_{\sigma}^{n+1}=\mathbf{e}_{F S Y}^{1}
$$

with

$$
\begin{aligned}
\mathbf{e}_{F S Y}^{1}= & -\delta t D_{f} Y G_{f}\left(\mathbf{P}^{n+1}-\mathbf{P}^{*}\right)-\delta t D_{f} Y C_{f \sigma}\left(\mathbf{U}_{\sigma}^{n+1}-\mathbf{U}_{\sigma}^{*}\right) \\
= & -\delta t D_{f} Y G_{f}\left(\mathbf{P}^{n+1}-\mathbf{P}^{*}\right)-D_{f} Y M_{f \sigma}\left(\mathbf{U}_{\sigma}^{n+1}-\mathbf{U}_{\sigma}^{*}\right) \\
& -\delta t D_{f} Y K_{f \sigma}\left(\mathbf{U}_{\sigma}^{n+1}-\mathbf{U}_{\sigma}^{*}\right) .
\end{aligned}
$$

Whereas the pressure term is of order $\mathcal{O}\left(\delta t^{q_{p}+2}\right)$, the interface term related to $M_{f \sigma}$ is $\mathcal{O}\left(\delta t^{q_{\sigma}+1}\right)$. Proceeding similarly for the structure equation, we get

$$
C_{\sigma f} \mathbf{U}_{f}^{n+1}+G_{\sigma} \mathbf{P}^{n+1}+\left(C_{\sigma \sigma}+N\right) \mathbf{U}_{\sigma}^{n+1}=\mathbf{b}_{\sigma}^{n+1}+\mathbf{e}_{F S Y}^{2},
$$

with

$$
\mathbf{e}_{F S Y}^{2}=-\delta t C_{\sigma f} Y G_{\sigma}\left(\mathbf{P}^{n+1}-\mathbf{P}^{*}\right)-\delta t C_{\sigma f} Y C_{f \sigma}\left(\mathbf{U}_{\sigma}^{n+1}-\mathbf{U}_{\sigma}^{*}\right) .
$$

By expanding $C_{\sigma f}$ and $C_{f \sigma}$ we can see that the order of accuracy of the pressure terms is $\mathcal{O}\left(\delta t^{q_{p}+1}\right)$ and for the interface terms $\mathcal{O}\left(\delta t^{q_{\sigma}}\right)$.

According to the previous considerations, a first order FSY scheme should involve a zeroth order pressure approximation and a first order interface velocity approximation. In our numerical experiments for the first order FSY scheme we use $\mathbf{P}^{*}=\mathbf{P}^{n}$ and $\mathbf{U}_{\sigma}^{*}=\mathbf{U}_{\sigma}^{n}$, as for the first order PIC scheme. Again, the error related to the pressure in this case is second order in time, but the one of the interface velocity is only first order.

6. The pressure-interface system. At every time step (or predictor-corrector iteration), all of the methods presented in section 3 evaluate the velocity field (inner nodes) decoupled from the coupled structure and pressure fields, for both intermediate and end-of-step velocity.

Let us make some comments about how to solve the pressure-interface velocity problem, which has the form:

$$
\left(\begin{array}{ll}
T_{p p} & T_{p \sigma} \\
T_{\sigma p} & T_{\sigma \sigma}
\end{array}\right)\left(\begin{array}{l}
\mathbf{P}^{n+1} \\
\mathbf{U}_{\sigma}^{n+1}
\end{array}\right)=\left(\begin{array}{l}
\mathbf{g}_{p} \\
\mathbf{g}_{\sigma}
\end{array}\right)
$$

where the force term depends on the method adopted and involves the intermediate velocity $\widetilde{\mathbf{U}}_{f}^{n+1}$. The added-mass effect can appear only in (6.1), which is much smaller than the original FSI system. The coupling problem involves the solution of $T$. For 
a sufficient condition on the nonsingularity of matrix $T$, see [23]. Matrix $T$ is illconditioned, with condition number $\kappa(T)=C_{T} h^{-2}$. An iterative solver, e.g., GMRES or BiCG, applied to the nonpreconditioned system (6.1) will exhibit slow convergence. Then a good preconditioner is mandatory. In the following we present two different approaches, even though other methods, e.g., multigrid, could be considered. The efficient solution of this system requires further research and will be the object of a forthcoming paper.

6.1. Losing modularity. Modularity is the property of a solver to consist of separated modules, and it is typical of the so-called partitioned procedures, which solve the fluid and the structure with two different codes.

A first and natural approach to solve system (6.1) would consist of building the system matrix $T$ and solving it by a preconditioned Krylov method. This approach, which involves the loss of modularity, is feasible only when the mass matrix is diagonal. In case of using nonmatching grids and a $L^{2}$-projection, the inverse mass matrix that will appear should be diagonal, too. Once matrix $T$ is assembled, we are able to use a classical preconditioner (such as $I L U(q)$ ) together with an iterative solver. When the mass matrix is not diagonal, the assembling of the Schur complements is not affordable.

6.2. Keeping modularity. When solving system (6.1) the key point is how to choose a good preconditioner in order to keep modularity for a Krylov iterative solver such as GMRES. This question is not new in FSI and is the seed of the added-mass effect. In [13], a preconditioned GMRES is used for solving every tangent system of the Newton method.

First of all, let us write the FSI problem (6.1) as an interface equation. This can be done by means of the Schur complement (the discrete version of the Steklov-Poincaré operator):

$$
\left(T_{\sigma \sigma}-T_{\sigma p} T_{p p}^{-1} T_{p \sigma}\right) \mathbf{U}_{\sigma}^{n+1}=\mathbf{g}_{\sigma}-T_{\sigma p} T_{p p}^{-1} \mathbf{g}_{p} .
$$

Also in this case, the system matrix is ill-conditioned, with a condition number of order $h^{-1}$. Thus, an optimal preconditioner must be used in (6.2). In order to keep modularity, this preconditioner can involve only structure (or fluid) terms. A classical choice is to take $N$ as a preconditioner of $\left(T_{\sigma \sigma}-T_{\sigma p} T_{p p}^{-1} T_{p \sigma}\right)$. This is the so-called Dirichlet-Neumann method. It can be proved that this method is optimal with respect to $h$ (see [27]). The preconditioned system is

$$
\begin{aligned}
\left(I+N^{-1} C_{\sigma \sigma}-\delta t N^{-1} C_{\sigma f} M_{f f}^{-1} C_{f \sigma}-N^{-1} T_{\sigma p} T_{p p}^{-1} T_{p \sigma}\right) & \mathbf{U}_{\sigma}^{n+1} \\
= & N^{-1} \mathbf{g}_{\sigma}-N^{-1} T_{\sigma p} T_{p p}^{-1} \mathbf{g}_{p} .
\end{aligned}
$$

Even though the spectral properties of matrix $N^{-1} T_{\sigma p} T_{p p}^{-1} T_{p \sigma}$ are mesh-size-independent, the spectral radius depends on a relationship between structure and fluid physical parameters, becoming ill-conditioned when the added-mass effect is critical.

The convergence properties of Richardson or GMRES procedures do depend on the spectral properties of $N^{-1} T_{\sigma p} T_{p p}^{-1} T_{p \sigma}$. When the weight of this matrix is small, the convergence properties of iterative procedures are good, while the convergence is slow or (for Richardson iterations) impossible in the presence of a strong added-mass effect.

By using a matrix-free iterative solver, we must evaluate a matrix-vector product at every iteration. Given a test vector $\mathbf{Z}$, we have to compute:

$$
N^{-1}\left(T_{\sigma \sigma}-T_{\sigma p} T_{p p}^{-1} T_{p \sigma}\right) \mathbf{Z}
$$


that is, the solution $\mathbf{W}$ of

$$
N \mathbf{W}=\left(T_{\sigma \sigma}-T_{\sigma p} T_{p p}^{-1} T_{p \sigma}\right) \mathbf{Z} .
$$

We can rewrite this system as

$$
\begin{aligned}
T_{p p} \mathbf{R} & =T_{p \sigma} \mathbf{Z}, \\
N \mathbf{W} & =N \mathbf{Z}+\left(C_{\sigma \sigma}-\delta t C_{\sigma f} M_{f f}^{-1} C_{f \sigma}\right) \mathbf{Z}-T_{\sigma p} \mathbf{R} .
\end{aligned}
$$

From (6.4), it is easily grasped why this preconditioner has been called DirichletNeumann. At the first step, where we evaluate the auxiliary array $\mathbf{R}$ (with the dimension of the pressure array), we are solving the pressure Schur complement associated to a Dirichlet fluid problem. The second step consists of a Neumann structure problem with the updated value of the pressure. Then we are keeping modularity, and appropriate solvers can be used separately for the solution of every field (pressure and structure). Let us make a further comment about how to solve the fluid problem

$$
T_{p p} \mathbf{R}=-\delta t D_{f} M_{f f}^{-1} G_{f} \mathbf{R}=T_{p \sigma} \mathbf{Z} .
$$

Again, we can use a matrix-free iterative solver and avoid the assembling of the matrices involving the inverse mass matrix. Anyway, it is much more appealing in terms of CPU cost to build $T_{p p}$ with a nondiagonal matrix and solve (6.5) by using an appropriate solver.

7. Numerical experiments. The test problem we have considered is the twodimensional $(2 d)$ benchmark proposed in [14]. It is a simplified blood flow problem which couples the $2 d$ incompressible Navier-Stokes equations for the fluid with the generalized string model $(1 d)$ with absorbing boundary conditions to describe the motion of the artery wall. The initial domain, obtained by intersecting a portion of blood flow vessel with a plane, is a rectangle of height $H=1 \mathrm{~cm}$ and length $L=6 \mathrm{~cm}$, whose upper and lower edges are deformable in the vertical direction. The goal is to simulate the deformation of the boundaries as the pressure pulse coming from the heart propagates in the artery.

The fluid and structure physical parameters used in the simulation have been listed in Table 7.1. These parameters have been chosen in the physiological range for a human body. Note that the values of $\rho_{s}$ and $\rho_{f}$ are very close. As a consequence, the added-mass effect is important. On the inflow section we impose the following Neumann boundary condition:

$$
\boldsymbol{\sigma}_{\text {in }}^{f}=-\frac{P_{\text {in }}}{2}\left[1-\cos \left(\frac{\pi t}{2.5 \cdot 10^{-3}}\right)\right] \mathbf{n},
$$

while on the outflow section a homogeneous Neumann condition has been imposed. The amplitude $P_{\text {in }}$ of the pressure pulse has been taken equal to $2 \cdot 10^{4}$ dynes $/ \mathrm{cm}^{2}$,

TABLE 7.1

Fluid and structure physical properties for the numerical test.

\begin{tabular}{|l|l|}
\hline Fluid density: $\rho_{f}=1.0 \mathrm{gr} / \mathrm{cm}^{3}$ & Fluid viscosity: $\mu=0.035$ poise \\
\hline Structure density: $\rho_{s}=1.1 \mathrm{gr} / \mathrm{cm}^{3}$ & Wall thickness: $h=0.1 \mathrm{~cm}$ \\
\hline Young modulus: $E=7.5 \cdot 10^{5}$ dynes $/ \mathrm{cm}^{2}$ & Viscoelastic parameter: $\gamma=10^{-1}$ dyne $\cdot \mathrm{s}$ \\
\hline Shear modulus: $G=2.5 \cdot 10^{5}$ dynes $/ \mathrm{cm}^{2}$ & Poisson coefficient: $\nu=0.5$ \\
\hline
\end{tabular}




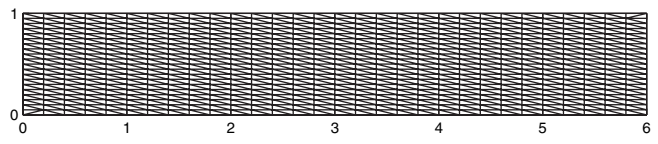

FIG. 7.1. Elliptic mesh used for the simulations.

and the time duration of the pulse is $5 \mathrm{~ms}$. We solve the problem over the time interval $[0,0.012] \mathrm{s}$.

We choose a conforming space discretization between fluid and structure: $\left(\mathbb{P}_{1}\right.$ iso $\left.\mathbb{P}_{2}\right)$ - $\mathbb{P}_{1}$ finite elements for the fluid and $\mathbb{P}_{1}$ finite elements for the structure. We have solved the problem with the algorithms described in section 3 on the elliptic mesh of $31 \times 21 \mathbb{P}_{1}$ fluid nodes $\left(2501 \mathbb{P}_{1}\right.$ iso $\mathbb{P}_{2}$ nodes $)$ shown in Figure 7.1.

7.1. Semi-implicit procedures. In Figure 7.2, we check the good behavior of a first order semi-implicit algorithm by evaluating its order of convergence in time. In order to do that, we solve the monolithic implicit scheme for $\delta t=10^{-6}$. The corresponding solution will be considered as exact.

We compare the solution of the first order semi-implicit monolithic method, computed on the mesh of Figure 7.1 for a sequence of decreasing time steps $(\delta t=$ $4 \cdot 10^{-4}, 2 \cdot 10^{-4}, 10^{-4}, 5 \cdot 10^{-5}$, and $4 \cdot 10^{-5}$ ) with the exact solution. In Figure 7.2 , we report the $L^{2}$-error on the fluid velocity, pressure, and structure displacement at time $t=10 \mathrm{~ms}$. In all cases, the method exhibits a first order of accuracy in time. Besides that, the semi-implicit method has remained stable.

7.2. PIC and FSY accuracy. The next step is to evaluate the convergence of the inexact factorization techniques designed in this article.
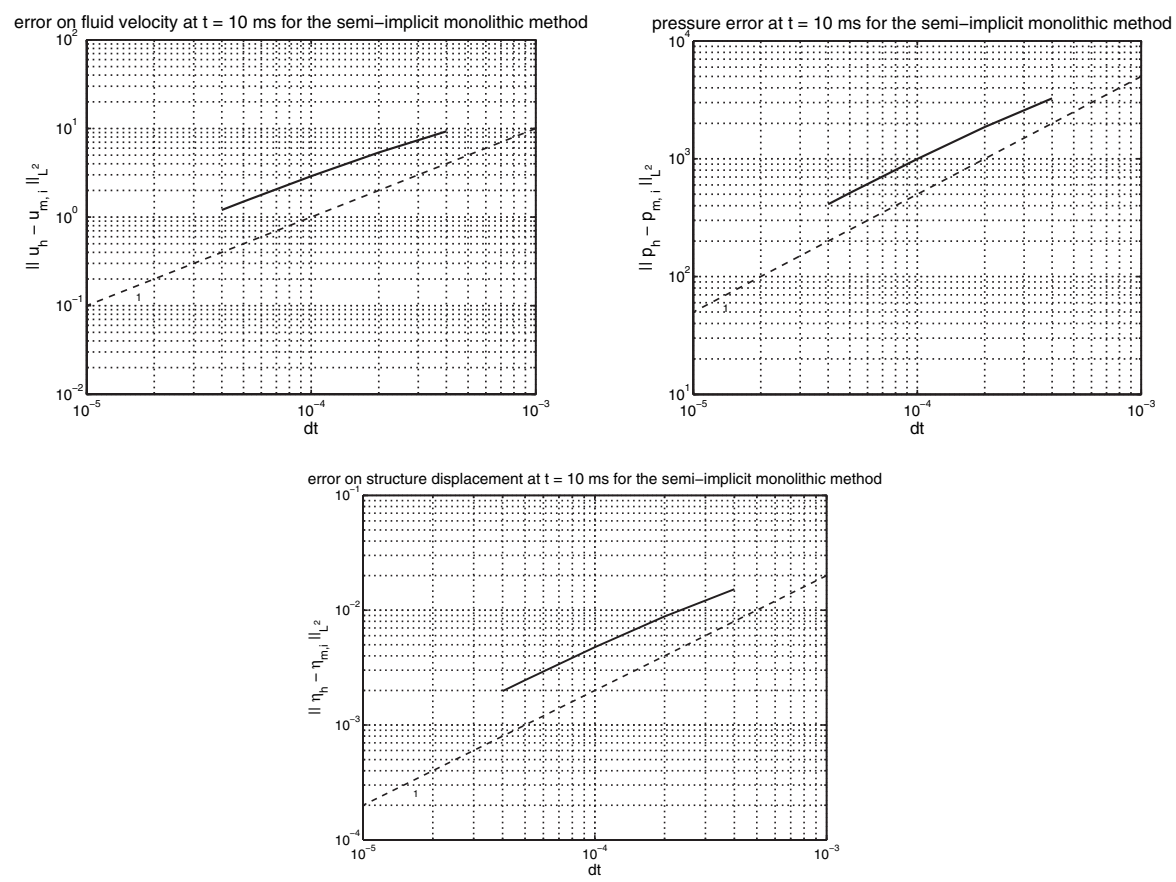

FIG. 7.2. Convergence of the semi-implicit monolithic method to the implicit monolithic one. The dashed line in each graph indicates slope 1. 

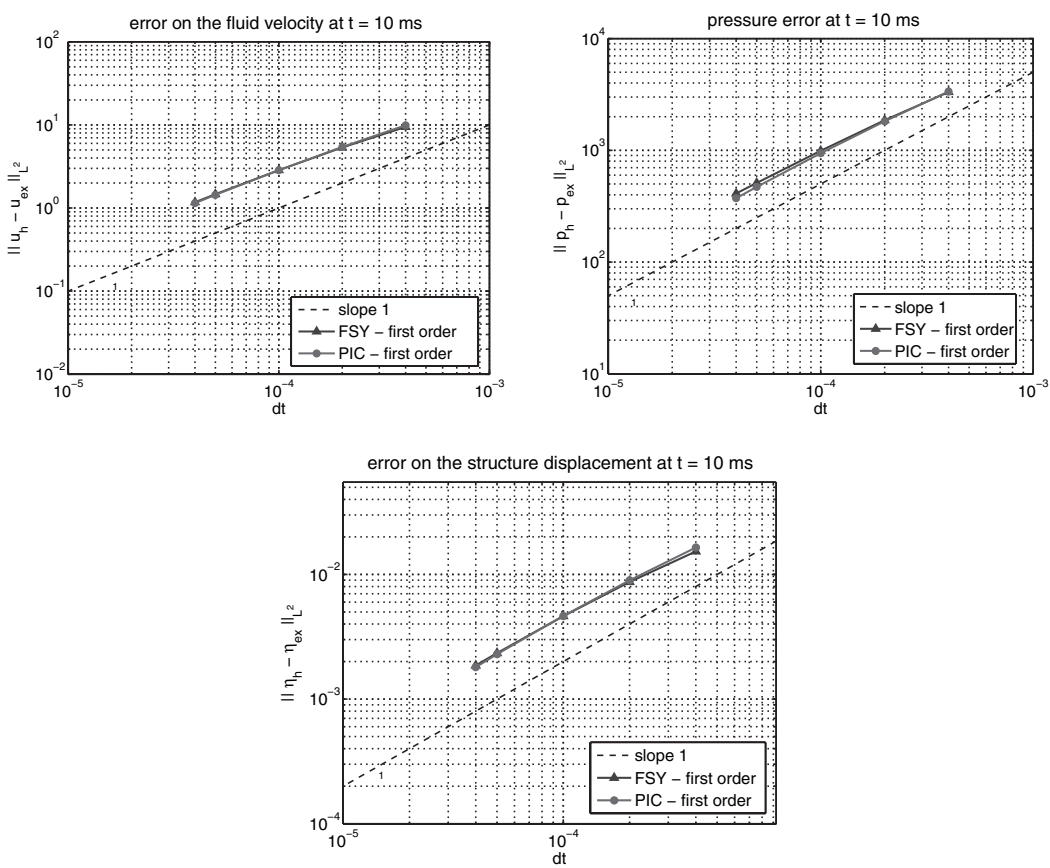

FIG. 7.3. Convergence of the first order FSY and PIC schemes to the monolithic semi-implicit method.

We solve the test problem with the first order FSY and PIC schemes (with first order predictions for pressure and interface velocity in the incremental FSI system) for the same sequence of time steps, always on the mesh of Figure 7.1. The FSI nonlinearity is treated in an explicit way by using semi-implicit schemes.

We compute the solution of the semi-implicit monolithic algorithm on the same mesh but with time step $\delta t=10^{-6}$ : We will address to this solution as the exact solution. We compare the solutions computed by the FSY and PIC methods at the different time steps with this exact solution. Figure 7.3 shows the error on the fluid velocity, pressure, and the structure displacement at time $t=10 \mathrm{~ms}$, all evaluated in the $L^{2}$-norm. As it was expected, we recover a linear convergence for both methods.

7.3. Convergence of predictor-corrector methods. The target is to analyze the convergence properties of predictor-corrector iterations with respect to the addedmass effect.

As commented in section 3.3, since the pressure and structure problems remain coupled after the inexact factorization, the convergence of this method towards the monolithic solution must be independent of the added-mass effect.

We have plotted the average number of predictor-corrector iterations (in time) for different values of the structure density: $\rho_{s}=500,100,50,10,5$, and $1 \mathrm{~g} / \mathrm{cm}^{3}$. We have performed this test for two different time step values. Figure 7.4 shows that the average number of predictor-corrector subiterations keeps almost constant for all of the values of $\rho_{s}$ in both cases.

7.4. The added-mass effect and the pressure-interface system. The pressure-interface velocity system couples fluid and structure problems. In section 6 we have discussed some possible alternatives for the solution of this linear system depending on modularity. 


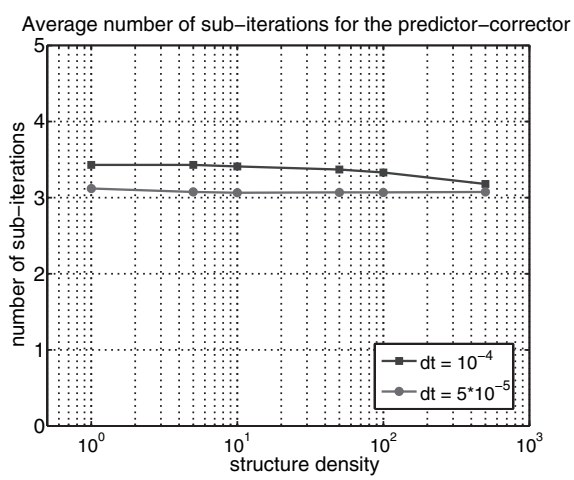

FIG. 7.4. Average subiterations of the predictor-corrector method as the structure density varies, for different time steps.
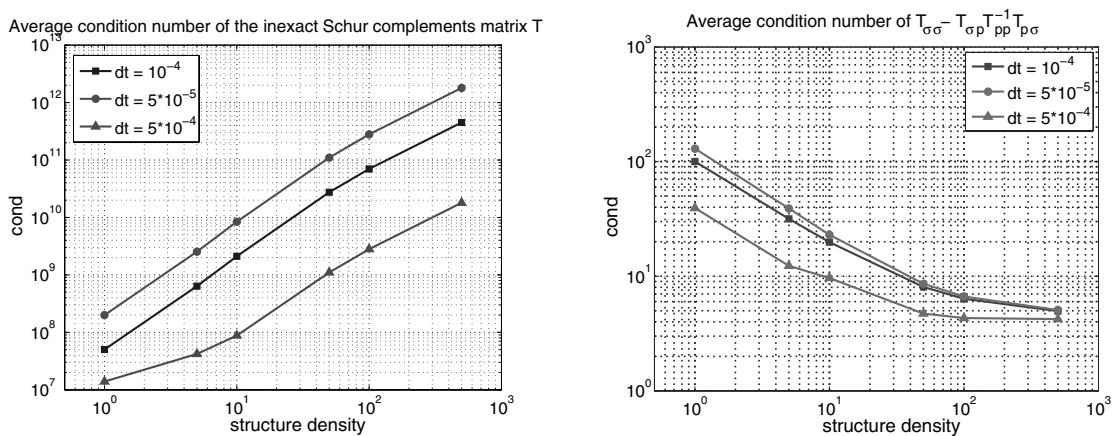

FIG. 7.5. Average conditioning number for the inexact Schur complement matrices and its Schur complement for different structure densities and time steps.

We want to evaluate how complicated it is to solve this system with respect to the added-mass effect. Again, we have solved the FSI test problem by using different values of the structure density $\left(\rho_{s}=500,100,50,10,5\right.$, and $\left.1 \mathrm{~g} / \mathrm{cm}^{3}\right)$ and different time steps $\left(\delta t=5 \cdot 10^{-4}, 10^{-4}\right.$, and $\left.5 \cdot 10^{-5}\right)$. We have evaluated the condition number of the system matrix $T$, which involves the loss of modularity. In Figure 7.5, we observe that the condition number of $T$ decreases with respect to the added-mass effect. Therefore, the solution of the fluid-structure system (6.1) is easier when the added-mass effect is more important.

On the other hand, we have evaluated the interface system matrix. As expected, due to the fact that this matrix is related to the interface problem, its condition number is much smaller. Moreover, the behavior with respect to the added-mass effect is opposite to the one for $T$ : The condition number of this interface matrix increases when $\rho_{s}$ approaches $\rho_{f}$.

As a conclusion, to lose modularity can be appealing when solving problems where the added-mass effect is critical. We also notice that, by keeping $\rho_{s}$ fixed, in both cases the average condition number increases as the time step decreases.

7.5. Qualitative results. In this section, we show average quantities computed on some section of the artery corresponding to the position $z_{i}=i \cdot h$, with $i=0, \ldots, 30$ and $h=0.2 \mathrm{~cm}$. We calculated the diameter of the artery, the average pressure, and the flux at each time step by using different strategies and numerical parameters. 

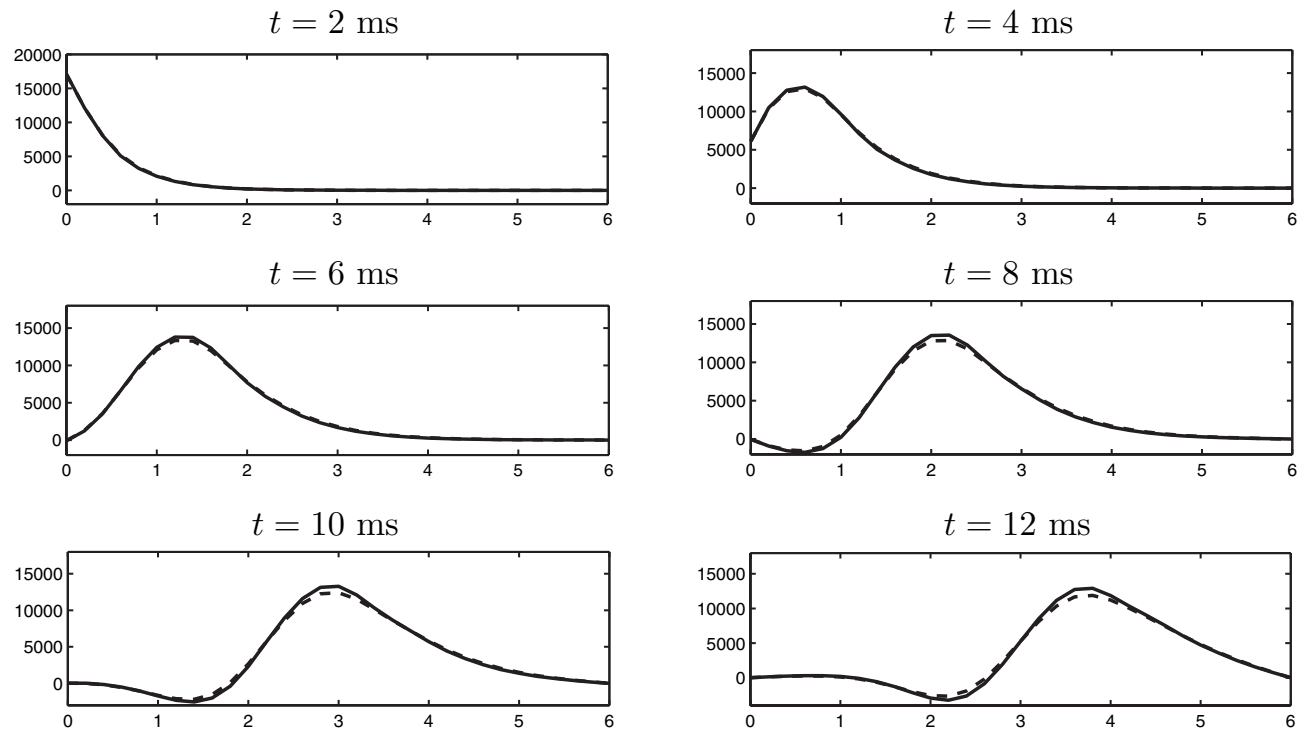

FIG. 7.6. Average pressure profiles computed with the first order FSY method for $\delta t=10^{-4}$ (dashed line) and for $\delta t=10^{-6}$ (solid line). Comparison at different time levels.
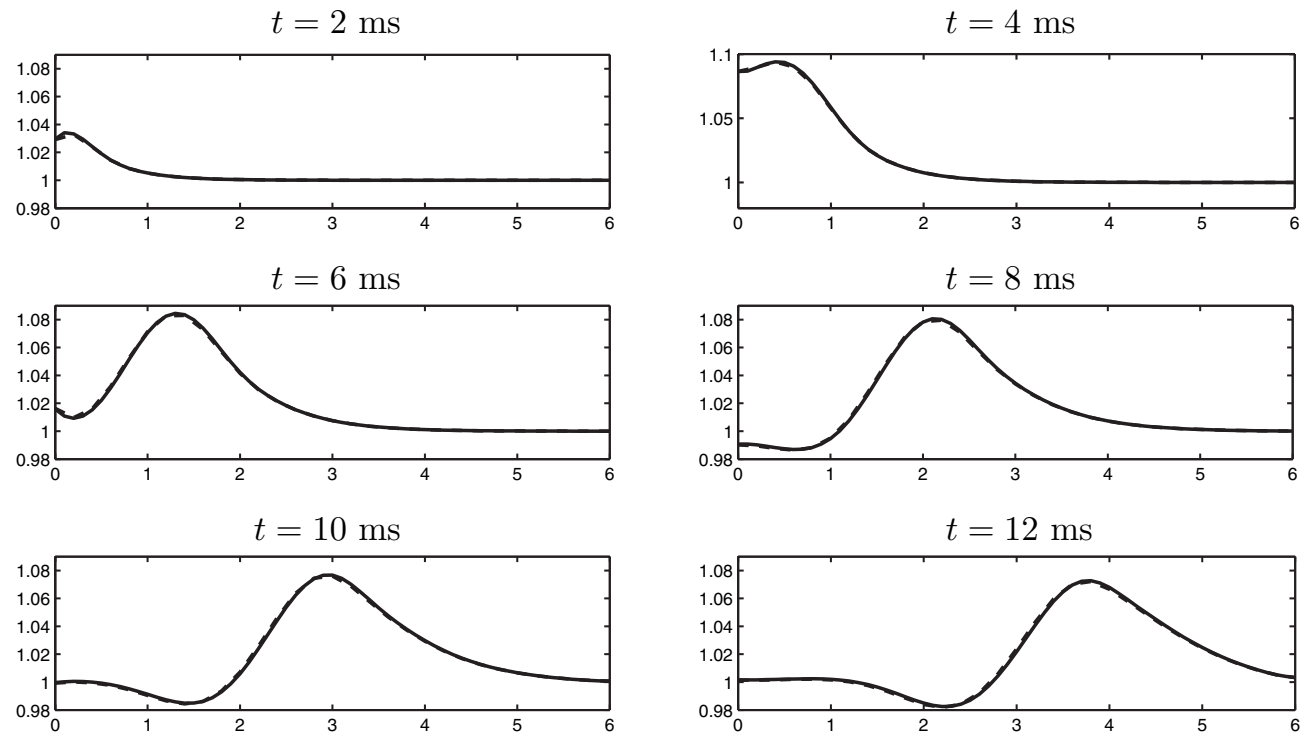

FIG. 7.7. Diameter of the artery section along its axis computed with the first order PIC method on the coarse mesh (dashed line) and on the fine mesh (solid line). Comparison at different time levels.

In Figure 7.6, we report the comparison between the average pressure profiles computed every 2 milliseconds with the first order FSY scheme on the mesh of Figure 7.1 but with two different time steps $\left(\delta t=10^{-4}\right.$ and $\left.\delta t=10^{-6}\right)$. As expected, for larger time step values, the solution is slightly more dissipative.

In order to evaluate the influence of the spatial discretization on the numerical solution, we compare in Figure 7.7 the diameter of the artery section calculated with 

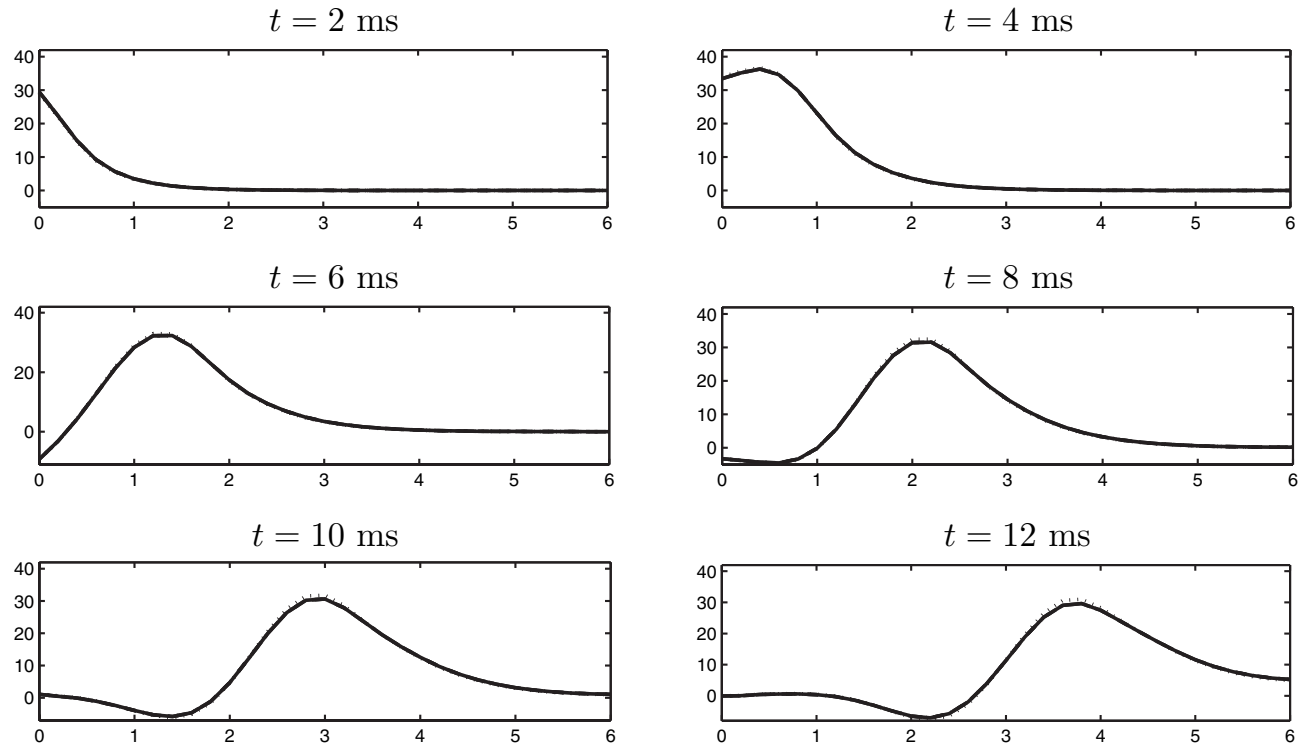

FIG. 7.8. Flow rate profiles at different time levels: comparison between the solution of first order FSY (dashed line), first order PIC (solid line), predictor-corrector (dashed-dotted line) methods, all for $\delta t=5 \cdot 10^{-5}$, and the "exact" solution (dotted line).
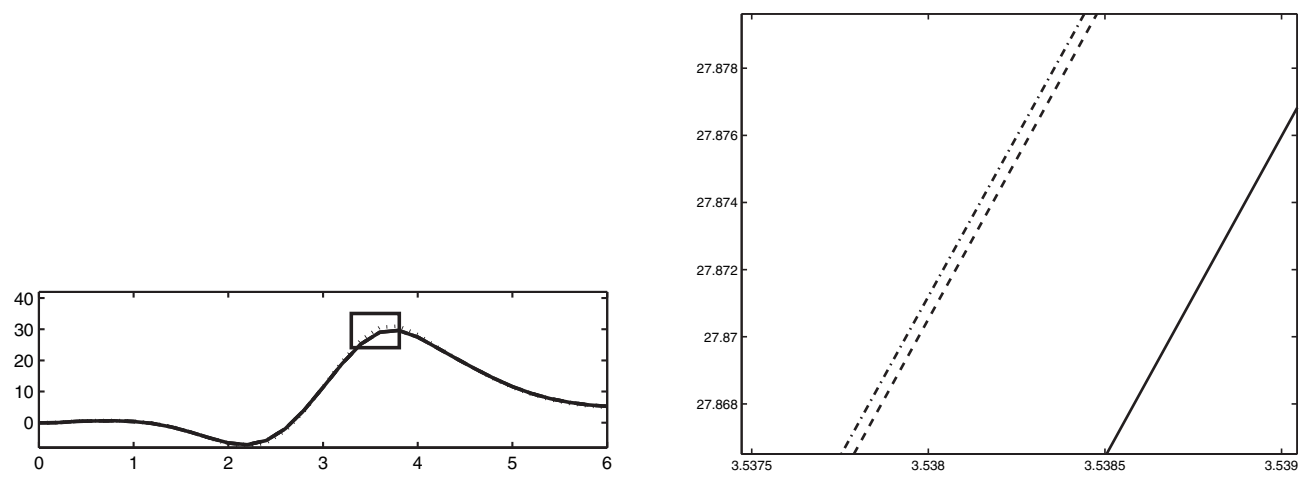

FIG. 7.9. On the left, the rectangle indicates the zoom area on the flow rate profiles for $t=$ $12 \mathrm{~ms}$. On the right, there is the zoom.

the first order PIC scheme on two different meshes: a coarse one $\left(31 \times 21 \mathbb{P}_{1}\right.$ fluid nodes) and a fine one $\left(61 \times 26 \mathbb{P}_{1}\right.$ fluid nodes $)$. In both cases the time step is $\delta t=10^{-4}$. We notice that the solution for the fine grid is slightly faster than the one computed on the coarse grid.

Finally, we compare, in a qualitative way, the flow rate of the monolithic scheme against those of PIC, FSY, and predictor-corrector methods for $\delta t=5 \cdot 10^{-5}$. In all cases we consider semi-implicit procedures. We notice from Figure 7.8 that the difference between the flow rate profiles associated to all of these solutions is very slight. Figure 7.9 shows a zoom of the flow rate profiles at $t=12 \mathrm{~ms}$.

8. Conclusions. In this work, we have focused on the numerical simulation of the FSI problems characterized by a strong added-mass effect. In such situations, good stability properties and low computational costs are shown by a semi-implicit 
coupling method introduced in [12]. The basic idea behind it is to couple implicitly the pressure stress to the structure, while the nonlinearity due to convection and the geometrical nonlinearities are treated explicitly. In [12], the implicit-explicit splitting is performed through a Chorin-Temam scheme for the fluid.

In this paper, we have proposed new schemes based on the inexact factorization of the linearized fluid-structure system; i.e., the procedure is split into explicit and implicit steps at the algebraic level. Two different methods have been designed: pressure-interface correction and fluid-structure Yosida. In both cases, the perturbation error has been analyzed and the convergence properties of the methods have been checked through numerical experiments. In the simulation of a pressure pulse propagation in a blood flow vessel, the methods remained stable for a wide range of discretization and physical parameters. Qualitative results have turned out to be very similar to those achieved with the monolithic system.

We have also proposed predictor-corrector methods that use inexact factors as preconditioners. The best feature of these procedures is that predictor-corrector iterations are independent of the added-mass effect. The solution of these methods converges to the one of the fully implicit monolithic system without introducing any perturbation. Therefore, these schemes are very well-suited when there is an interest on implicit fluid-structure solutions. In this case, we can also consider one-loop algorithms, where nonlinearity and predictor-correction iterations are performed with only one loop.

The next step will concern the implementation of second order (in time) PIC and FSY methods and the application of the algorithms presented here to threedimensional problems. These more realistic cases would also enable us to evaluate the computational cost reduction allowed by the methods that we have introduced in this paper. We shall also investigate the applicability of other algebraic fractional-step methods, such as Yosida scheme modifications called Yosida3 and Yosida4 [16].

Acknowledgements. We thank Dr. Fabio Nobile and Dr. Christian Vergara for helpful discussions and comments.

\section{REFERENCES}

[1] S. BADIA AND R. Codina, On some fluid-structure iterative algorithms using pressure segregation methods. Application to aeroelasticity, Internat. J. Numer. Methods Engrg., 72 (2007), pp. 46-71.

[2] P. Causin, J.-F. Gerbeau, and F. Nobile, Added-mass effect in the design of partitioned algorithms for fluid-structure problems, Comput. Methods Appl. Mech. Engrg., 194 (2005), pp. 4506-4527.

[3] M. Cervera, R. Codina, and M. Galindo, On the computational efficiency and implementation of block-iterative algorithms for nonlinear coupled problems, Engrg. Comput., 13 (1996), pp. 4-30.

[4] A.J. Chorin, Numerical solution of the Navier-Stokes equations, Math. Comp., 22 (1968), pp. $745-762$.

[5] R. Codina, Stabilized finite element approximation of transient incompressible flows using orthogonal subscales, Comput. Methods Appl. Mech. Engrg., 191 (2002), pp. 4295-4321.

[6] R. CODINA AND S. BADIA, On some pressure segregation methods of fractional-step type for the finite element approximation of incompressible flow problems, Comput. Methods Appl. Mech. Engrg., 195 (2006), pp. 2900-2918.

[7] R. Codina And O. Sото, Approximation of the incompressible Navier-Stokes equations using orthogonal-subscale stabilization and pressure segregation on anisotropic finite element meshes, Comput. Methods Appl. Mech. Engrg., 193 (2004), pp. 1403-1419.

[8] S. Deparis, M. Discacciati, G. Fourestey, and A. Quarteroni, Fluid-structure algorithms based on Steklov-Poincaré operators, Comput. Methods Appl. Mech. Engrg., 195 (2006), pp. $5797-5812$. 
[9] S. Deparis, M. Discacciati, And A. Quarteroni, A domain decomposition framework for fluid-structure interaction problems, in Proceedings of the Third International Conference of Computational Fluid Dynamics (ICCFD3), C. Groth and D.W. Zingg, eds., Springer, New York, 2006, pp. 41-58.

[10] S. Deparis, M.A. Fernández, And L. Formaggia, Acceleration of a fixed point algorithm for fluid-structure interaction problems, M2AN Math. Model. Numer. Anal., 37 (2003), pp. 601-616.

[11] H.C. Elman, Preconditioners for saddle point problems arising in computational fluid dynamics, Appl. Numer. Math., 43 (2002), pp. 75-89.

[12] M.A. Fernández, J.F. Gerbeau, and C. Grandmont, A projection semi-implicit scheme for the coupling of an elastic structure with an incompressible fluid, C. R. Math. Acad. Sci. Paris, 342 (2006), pp. 279-284.

[13] M.A. Fernández and M. Moubachir, A Newton method using exact Jacobians for solving fluid-structure coupling, Comput. \& Structures, 83 (2005), pp. 127-142.

[14] L. Formaggia, J.-F. Gerbeau, F. Nobile, and A. Quarteroni, On the coupling of $3 D$ an $1 D$ Navier-Stokes equations for flow problems in compliant vessels, Comput. Methods Appl. Mech. Engrg., 191 (2001), pp. 561-582.

[15] J.F. Gerbeau AND M. Vidrascu, Quasi-Newton algorithm based on a reduced model for fluid-structure interaction problems in blood flows, M2AN Math. Model. Numer. Anal., 37 (2003), pp. 631-647.

[16] P. Gervasio, F. Saleri, and A. Veneziani, Algebraic fractional step schemes with spectral methods for the incompressible Navier-Stokes, J. Comput. Phys., 214 (2006), pp. 347-365.

[17] M. HEIL, An efficient solver to the fully coupled solution of large-displacement fluid-structure interaction problems, Comput. Methods Appl. Mech. Engrg., 193 (2004), pp. 1-23.

[18] D. Loghin And A.J. WAThen, Schur complement preconditioners for the Navier-Stokes equations, Internat. J. Numer. Methods Fluids, 40 (2002), pp. 403-412.

[19] H.G. Matthies And J. Steindorf, Partitioned strong coupling algorithms for fluid-structure interaction, Comp. \& Structures 81 (2003), pp. 805-812.

[20] D.P. MoK AND W.A. WAll, Partitioned analysis schemes for the transient interaction of incompressible flows and nonlinear flexible structures, in Trends in Computational Structural Mechanics, K. Schweizerhof, W.A. Wall, and K.U. Bletzinger, eds., CIMNE, Barcelona, 2001.

[21] D.P. MoK, W.A. WALL, AND E. RAmM, Accelerated iterative substructuring schemes for instationary fluid-structure interaction, in Computational Fluid and Solid Mechanics, K.J. Bathe, ed., Elsevier, New York, 2001.

[22] F. NoBiLe, Numerical Approximation of Fluid-Structure Interaction problems with application to Haemodynamics, Ph.D. thesis, École Polytechnique Fédérale de Lausanne, 2001.

[23] A. Quaini And A. Quarteroni, A semi-implicit approach for fluid-structure interaction based on an algebraic fractional step method, Math. Models Methods Appl. Sci., 17 (2007), pp. 957-983.

[24] A. Quarteroni, G. Sacchi Landriani, and A. Valli, Coupling viscous and inviscid Stokes equations via a domain decomposition method for finite elements, Numer. Math., 59 (1991), pp. 831-859.

[25] A. Quarteroni, F. Saleri, and A. Veneziani, Analysis of the Yosida method for the incompressible Navier-Stokes equations, J. Math. Pures Appl., 78 (1999), pp. 473-503.

[26] A. Quarteroni, F. Saleri, and A. Veneziani, Factorization methods for the numerical approximation of Navier-Stokes equations, Comput. Methods Appl. Mech. Engrg., 188 (2000), pp. 505-526.

[27] A. Quarteroni and A. Valli, Domain Decomposition Methods for Partial Differential Equations, Oxford Science, London, 1999.

[28] P. Le Tallec and J. Mouro, Fluid structure interaction with large structural displacements, Comput. Methods Appl. Mech. Engrg., 190 (2001), pp. 3039-3067.

[29] R. Temam, Sur l'approximation de la solution des équations de Navier-Stokes par la méthode des pas fractionaires (I), Arch. Ration. Mech. Anal., 32 (1969), pp. 135-153.

[30] T.E. TezduYar, Finite element methods for fluid dynamics with moving boundaries and interfaces, Arch. Comput. Methods Engrg., 8 (2001), pp. 83-130.

[31] S. Turek, Efficient Solvers for Incompressible Flow Problems, Lect. Notes Comput. Sci. Eng. 6, Springer, New York, 1999.

[32] A. Veneziani, A Mathematical and Numerical Modeling of Blood Flow Problems, Ph.D. thesis, University of Milan, 1998. 